\title{
Criminologie
}

\section{Politiques de rage et narcissisme malin}

\section{Jodi M. Lane et Stephen A. Kent}

Volume 41, numéro 2, automne-hiver 2008

Les organisations dites sectes, les lois et la société

URI : https://id.erudit.org/iderudit/019435ar

DOI : https://doi.org/10.7202/019435ar

Aller au sommaire du numéro

Éditeur(s)

Les Presses de l'Université de Montréal

ISSN

0316-0041 (imprimé)

1492-1367 (numérique)

Découvrir la revue

Citer cet article

Lane, J. M. \& Kent, S. A. (2008). Politiques de rage et narcissisme malin. Criminologie, 41(2), 117-155. https://doi.org/10.7202/019435ar

\section{Résumé de l'article}

Dans cet article, le trouble de la personnalité connu sous le nom de " narcissisme malin » est présenté. Ce concept est par la suite utilisé pour expliquer la création, par le dirigeant d'un groupe, de politiques organisationnelles destinées à contrer les personnes qu'il considérait comme ennemies, mettant en évidence la rage narcissique à l'oeuvre. Notre argument est examiné à la lumière d'une étude de cas dans laquelle il est démontré que le dirigeant a tenté de discréditer les détracteurs du groupe, transposant sa rage narcissique dans des politiques organisationnelles que des membres loyaux adoptaient pour lui. À l'aide d'observations psychologiques de la personnalité du chef et, par la suite, en démontrant comment celle-ci est à la source de politiques et d'actions socialement déviantes, nous espérons encourager les criminologues à examiner d'autres groupes en appliquant des théories semblables.
Ce document est protégé par la loi sur le droit d'auteur. L'utilisation des services d’Érudit (y compris la reproduction) est assujettie à sa politique d'utilisation que vous pouvez consulter en ligne.

https://apropos.erudit.org/fr/usagers/politique-dutilisation/ 


\title{
Politiques de rage et narcissisme malin $^{1}$
}

\author{
Jodi M. Lane \\ Étudiante à la maîtrise \\ Département de sociologie \\ Université d'Alberta \\ jmlane1971@hotmail.com
}

\author{
Stephen A. Kent \\ Professeur \\ Département de sociologie et Département d'études religieuses \\ Université d'Alberta \\ steve.kent@ualberta.ca
}

RÉSUMÉ - Dans cet article, le trouble de la personnalité connu sous le nom de «narcissisme malin» est présenté. Ce concept est par la suite utilisé pour expliquer la création, par le dirigeant d'un groupe, de politiques organisationnelles destinées à contrer les personnes qu'il considérait comme ennemies, mettant en évidence la rage narcissique à l'œuvre. Notre argument est examiné à la lumière d'une étude de cas dans laquelle il est démontré que le dirigeant a tenté de discréditer les détracteurs du groupe, transposant sa rage narcissique dans des politiques organisationnelles que des membres loyaux adoptaient pour lui. À l'aide d'observations psychologiques de la personnalité du chef et, par la suite, en démontrant comment celle-ci est à la source de politiques et d'actions socialement déviantes, nous espérons encourager les criminologues à examiner d'autres groupes en appliquant des théories semblables.

Traduit de l'anglais par Grégoire Dodier et Pierre Drolet.

1. Les auteurs remercient les nombreux anciens membres de la Scientologie qui ont fourni des documents et des informations. Sans leur générosité, cet article n'aurait pu voir le jour. 


\section{Introduction}

Grandiosité ${ }^{2}$, élitisme (Wilson, 1970: 26-27; Atack, 1990: 181), des visions de transformation ${ }^{3}$ et des exigences de loyauté (Storr, 1996: 209; Lalich, 2004: 52, 90, 142, 241) sont communs chez de nombreux chefs religieux (Singer et Lalich, 1995: 8-10, 39-40) ${ }^{4}$, et ces caractéristiques sont sources d'inspiration pour les acolytes qui les intériorisent. Souvent, ces chefs surgissent et se font des disciples durant des périodes de perturbations sociales et culturelles (Cohn, 1970: 52; Kent, 1987c; 1989; 2001) et «dans des moments de détresse - qu'elle soit psychique, physique, économique, morale, religieuse ou politique» (Weber, 1922 [1968]: 1112; Glock, 1964). Cependant, parmi ces facteurs et d'autres éléments sociaux (tels que «la laïcisation, le pluralisme et la privatisation») qui font que des sectes sont créées (Clarke, 2006:

2. Au sujet de la grandiosité du gourou Maharaj Ji (chef de la Mission de la Lumière Divine) au début des années 1970, dont les plans de construire une cité divine étaient supposément si importants qu'ils devaient attirer l'attention des extra-terrestres, voir Kent, 2001 : 156. Au sujet du rêve et des plans du fondateur de la Scientologie, L. Ron Hubbard, de créer une cité internationale dans laquelle les scientologues auraient le monopole de la santé mentale, voir Kent, 1999: 154. Au sujet des plans grandioses de Rajneesh de créer l'«Homo Novus»-le nouvel humain qui serait au-delà du bien et du mal, libre des normes et des règles de la culture, mais qui parviendrait d'une manière ou d'une autre à vivre en paix et dans l'amour, voir Carter, 1990: 66. Au sujet de la déclaration de Dwight York, chef de la United Nuwaubian Nation of Moors (dans le comté de Putnam, en Géorgie), selon laquelle il était «l'incarnation de Dieu pour cette époque» (avant d'être condamné à 135 ans de prison pour abus sexuel d'enfants), voir Osinski, 2007: 68-69. Il existe beaucoup d'autres affirmations grandioses faites par des chefs de nouvelles religions et de groupes connexes.

3. Par exemple, «Une révélation de la divinité hindoue Shiva, qui devint la principale divinité à Aum [Shinrikyo], a amené [le fondateur Shoko] Asahara à se considérer comme une figure messianique qui devait conduire ses disciples à l'établissement d'une société idéale désignée sous le nom de Kingdom of Shambala (Mullins, 1997: 316). De la même façon, le révérend Sun Myung Moon de l'Église de l'Unification a rapporté «que le jour de Pâques, en 1936, Jésus lui est apparu et lui a révélé que Dieu l'avait choisi pour la mission d'établir Son Royaume des Cieux sur terre» (Barker, 1984: 28). Pendant qu'il était sur le mont Sion, en Israël, le chef de la Branch Davidian, David Koresh, a prétendu «que sept êtres angéliques lui étaient apparus et lui avaient expliqué les secrets des sept sceaux [dans le Livre des révélations]» (Newport, 2006: 183). D’une façon générale, «de nombreux chefs de cultes rapportent des expériences mystiques miraculeuses et de conversion qui les ont conduits sur la route du leadership religieux» (Deutsch, 1989: 148).

4. Nous ne voulons pas entreprendre un débat terminologique sur les termes secte, culte ou nouvelle religion et dans la présente étude, nous utilisons plus ou moins l'un ou l'autre de ces termes pour désigner des groupes non traditionnels qui véhiculent des messages non traditionnels contenant à divers degrés des affirmations surnaturelles. Nous ne voulons pas non plus argumenter à savoir lequel de ces termes décrit le mieux la Scientologie selon des définitions théoriques plus formelles. 
16-21), il faut mentionner la personnalité peu commune des fondateurs de groupes ${ }^{5}$. Il y a de fortes chances que les fondateurs sectaires soient animés de convictions profondes - une certitude et une assurance déterminées émanent de leur personnalité qui exprime le pouvoir et qui attire des disciples. L'imposante personnalité du début de la sociologie de la religion, Max Weber, appelle ces qualités «du charisme», mais des spécialistes contemporains des sciences sociales étiquettent quelquesunes de ces mêmes personnes comme des malades mentaux ou des personnes souffrant d'un déséquilibre de la personnalité (Deutsch, 1983 : 122-128; 1989: 156-257; Numbers et Numbers, 1992; Storr, 1996: 152-158; Lys, 2005; Raine, 2005). Les recherches effectuées sur la vie et les activités du fondateur du mormonisme, Joseph Smith, fournissent le meilleur exemple de ce double étiquetage. Quand il a élaboré son concept du charisme, Weber a explicitement mentionné Smith, bien que ce fût loin d'être d'une manière flatteuse. Dans la définition classique du concept (rédigée quelque part entre 1918 et l'année de sa mort, 1920), Weber a écrit:

Le terme «charisme» sera utilisé pour une certaine qualité de la personnalité individuelle en vertu de laquelle la personne est considérée comme extraordinaire et traitée comme pourvue de pouvoirs ou de qualités surnaturels, surhumains ou du moins explicitement exceptionnels. Ces qualités sont telles qu'elles ne sont pas accessibles à la personne ordinaire, mais qu'elles sont plutôt considérées comme d'origine divine ou exemplaires, et compte tenu de ces qualités, l'individu concerné est traité comme un «leader». (Weber, 1922 [1968]: 241)

5. Il y a eu, bien sûr, depuis le début du $\mathrm{xx}^{\mathrm{e}}$ siècle, des discussions théoriques au sujet de la relation entre la santé mentale et la fondation de nouvelles religions. The Varieties of Religious Experience (1902) de William James, demeure la plus connue de ces nombreuses études, mais plusieurs autres auteurs se sont lancés dans le débat entourant (par exemple) la santé mentale de George Fox en rapport avec l'origine des Quakers (voir Kent, 1987a; 1987b). Le modèle psychopathologique présenté par les sociologues William Sims Bainbridge et Rodney Stark (1979) est une tentative récente d'établir la corrélation entre l'état psychique des fondateurs de culte (c'est le nom attribué par les chercheurs) et les groupes qu'ils ont créés. Ce modèle considérait dans certains cas «la fondation de culte comme l'aboutissement d'une psychopathologie individuelle qui s'exprime dans un succès social» (Bainbridge et Stark, 1979: 173). Bien que d'une certaine façon le modèle biopsychosocial sur lequel s'appuie notre travail (Kent, 2006) partage des similarités avec la position de Bainbridge et Stark, nous considérons que le langage et les concepts de psychologie et de psychiatrie qu'ils emploient sont beaucoup trop imprécis, quand ils ne sont pas inexacts. Par exemple, ils parlent de L. Ron Hubbard dans un paragraphe au sujet de la «paranoïa classique et de la schizophrénie paranoïaque» (Bainbridge et Stark, 1979: 175). Bien que Hubbard ait montré des caractéristiques paranoïaques, il n'était à tout le moins sûrement pas schizophrène. 
Plusieurs phrases plus loin, il a ajouté: «Aux fins de la présente, il sera nécessaire de traiter une variété de différents types représentant des personnes comme pourvues de charisme dans le sens que [...] Un autre type est représenté par Joseph Smith, le fondateur du mormonisme, qui a pu être un escroc très sophistiqué (bien que cela ne puisse être établi hors de tout doute)» (Ibid.: 242, 1112).

L'auteur d'un livre récent sur Smith utilise cependant une approche très différente. Bien qu'il ne mette nullement en doute le charisme de Smith (Anderson, 1999: 236), le psychiatre et ex-mormon Robert D. Anderson a exposé l'argument convaincant que Smith présentait en fait un trouble appelé «narcissisme malin». Considérant que le narcissisme malin combinait les caractéristiques à la fois de la personnalité antisociale et narcissique, Anderson a écrit :

Un comportement moral pourrait se manifester dans certaines activités, et une mentalité d'exploiteur dans certaines autres pour des états moins graves de la maladie. Quelques individus peuvent ressentir certaines formes de culpabilité, d'inquiétude et de loyauté envers les autres. Ils pourraient être capables de planifier pour l'avenir. Ces expressions moins graves du narcissisme malin se caractérisent par la promiscuité sexuelle avec les disciples ou leur exploitation financière. Pourtant, dans d'autres occasions, les personnes atteintes font preuve d'honnêteté et de cohérence. Elles peuvent tenir les autres responsables de leurs problèmes et trouver des excuses à leurs difficultés.

Dans le cas de Joseph Smith, le fil conducteur de sa vie, qui a pour thème de se duper et de tromper les autres, n'est pas un fil, mais un câble d'acier. Rarement de telles caractéristiques n'ont-elles été si bien documentées. (Anderson, 1999: 230)

Pour appuyer le diagnostic de Joseph Smith, Anderson a examiné chez le prophète «ses conquêtes sexuelles sous le couvert de pratiques religieuses», «ses gestes malhonnêtes», le fait qu'il n'hésitait pas «à attribuer la responsabilité à des ennemis imaginaires» qui s'ingéraient dans son travail, qu'il «condamnait les victimes» qui avaient contracté une maladie et l'habitude qu'il avait de mettre sur le dos des autres «l'échec de son entreprise dans les affaires bancaires» (Ibid.: 231) ${ }^{6}$. Le

6. Même le plus connu des biographes de Smith, Fawn Brodie, a réalisé qu'il y avait quelque chose qui ne tournait pas rond chez le prophète. Elle a écrit par exemple: «Le lecteur du dimanche sera choqué par les supercheries de [Smith] - parfois maladroites, mais encore plus choquantes lorsqu'elles étaient faites d'habile façon - parce que Joseph pratiquait dans le domaine de la religion, domaine où l'honnêteté et l'intégrité devraient 
désir d'une personne de blesser ou d'anéantir ceux qu'elle considère comme des adversaires fait en sorte que son trouble de la personnalité est classé «malin». Si on le critique, ce type de narcissique «peut réagir avec dédain, rage ou par une contre-attaque provocante» (American Psychiatric Association, 2000b). Ces caractéristiques du narcissisme et du narcissisme malin sont présentes chez d'autres leaders charismatiques $^{7}$, dont l'un est le sujet de cette étude ${ }^{8}$.

Ce fondateur sectaire ayant un intérêt particulier pour nous est un autre leader qui présentait de nombreuses caractéristiques du narcissisme malin, L. Ron Hubbard (1911-1986), fondateur et «théologien» singulier de l'organisation idéologique connue sous le nom de Scientologie. Nous ne sommes certainement pas les premiers à avoir étudié Hubbard et à mentionner qu'il pourrait avoir souffert de névrose narcissique (voir Atack, 1990: 372; Kent, 2006: 347) ${ }^{9}$, mais cet article est la première tentative qui situe la structure de la personnalité de

sans doute compter pour quelque chose.» (Brodie, 1963: 84) Un peu plus loin, elle ajoute: «Et au début, il semble avoir atteint un équilibre interne qui lui aurait permis de poursuivre sa carrière avec une sincérité hautement compensée, mais néanmoins très réelle. Un sentiment de culpabilité persistant à cause des supercheries grâce auxquelles sa carrière prophétique a été lancée l'aurait certainement détruit éventuellement.» (Ibid.: 85). Le fait de voir Smith en tant que narcissique donne un sens nouveau à ces observations et à beaucoup d'autres au sujet du fondateur du mormonisme.

7. Pour des analyses sur des leaders charismatiques de sectes, de cultes et de nouvelles religions considérés comme des narcissiques ou des narcissiques malins, voir: Clark, 1988 (au sujet de Rajneesh); Kent, 2007 (au sujet d'Alexander d'Abonuteichos, dans l'antiquité); Krakauer, 2003 : 162, 303-307 (au sujet de l'intégriste homicide mormon Ronald Lafferty); Mascareñas de los Santos et Mascareñas Ruiz, 1997: 102-105 (au sujet du chef actuel de La Luz del Mundo, Samuel Joaquín Flores); la National Parole Board, 2003 : 3 (au sujet du criminel détenu Ivon Shearing, chef d'une secte de la Colombie-Britannique appelée Kabalarians); Sil, 1991 (au sujet du célèbre gourou indien Ramakrsna Paramahamsa). Pour des commentaires généraux sur des gourous et des chefs de secte considérés comme narcissiques, voir Storr, 1996: 208-211.

8. Nous devons faire remarquer que nous ne sommes pas les premiers chercheurs à établir des parallèles entre la vie de Smith et celle de Hubbard. Dans un article sur les «fraudes religieuses» dans l'Encyclopedia of White Collar and Corporate Crime (Barnhill, 2005), la première phrase se lit comme suit: «De Joseph Smith, qui a fondé le mormonisme après une révélation qui porte encore des accusations de canular à L. Ron Hubbard et aux scientologues enclins à intenter des poursuites, la religion et la fraude ont été inextricablement liées, soit à la suite de méfaits ou dans la perception des non-croyants et des sceptiques.» (Barnhill, 2005: 679) Notre article en arrive à la conclusion que les perceptions de fraude par les autres proviennent sans doute de la probabilité que les deux chefs aient démontré des comportements en rapport avec le narcissisme.

9. Dans une étude antérieure, un des auteurs (Kent) a affirmé: «Selon mes lectures, [Hubbard] était sans doute un individu qui présentait une combinaison des caractéristiques de la paranoïa et du narcissisme.» (Kent, 2006: 347) Le modèle narcissique que nous utilisons ici considère cependant la paranoïa comme un aspect du narcissisme. 
Hubbard dans les limites de la documentation universitaire actuelle sur cette maladie. De plus, nous allons montrer que Hubbard présentait des traits d'une forme particulière de la pathologie, le narcissisme malin, vu la façon dont il réagissait devant les personnes perçues par lui comme adversaires, et que ces réactions personnelles expliquent les politiques organisationnelles de représailles et de vengeance de la Scientologie. Essentiellement, l'atmosphère institutionnelle de la Scientologie reflète en grande partie le narcissisme et la rage narcissique maligne de Hubbard. Nous appuyons cette affirmation sur des informations concernant la vie de l'homme ainsi qu'à l'aide de nombreux documents de première importance qui exposent les politiques de la Scientologie qu'il a conçues pour réprimer ou faire taire ses détracteurs et ses ennemis.

\section{Narcissisme}

Le terme narcissisme remonte à la mythologie grecque et au poète Ovide (43 avant notre ère à 17 [?] après notre ère). Des représentations du concept ont été découvertes dans les œuvres d'art, et le terme a été utilisé dans la littérature à travers les siècles, mais ce n'est que vers la toute fin $\mathrm{du} \mathrm{xIX}^{\mathrm{e}}$ siècle qu'il a été introduit dans la documentation en psychiatrie (Kernberg, 1998: 31; Ronningstram, 2005: 4-5). Un psychiatre formé à l'écoute psychanalytique, Otto Kernberg, a introduit le concept du narcissisme malin au début des années 1980, pour les personnes dont «l'estime de soi augmente et dont la grandiosité est confirmée quand elles peuvent exprimer de l'agressivité envers elles-mêmes et les autres» (Kernberg, 1984: 257, 290-297; 1998: 44). Ces observations et d'autres données de la psychanalyse conviennent à l'approche plus descriptive et empirique (par opposition à l'analyse intrapsychique) que le Manuel diagnostique et statistique des troubles mentaux a adopté après son édition de 1980 (Ronningstam et Gunderson, 1990; Wilson, 1993; Ronningstram, 2005: 11, 16-19, 22-25). Cependant, une contribution psychanalytique à la compréhension actuelle du narcissisme peut être trouvée dans un résumé récent des résultats de la recherche sur la maladie et elle est réalisée par l'un des chercheurs les plus en vue dans le domaine:

Au-delà du [Manuel diagnostique et statistique des troubles mentaux], il existe maintenant un soutien à la fois clinique et empirique pour définir l'essentiel de la pathologie narcissique autour de quatre principaux éléments de 
fonctionnement: la régulation de l'estime de soi; la régulation affective; les relations interpersonnelles; le fonctionnement du surmoi [c'est-à-dire, les systèmes de moralité intériorisés]. De plus, plusieurs comptes-rendus soutiennent l'existence de trois sous-types de [névrose narcissique]: (1) le type arrogant, inconscient, non réprimé; (2) le type timide, hypervigilant, secret; (3) le type narcissique psychopathique. (Ronningstram, 2005: 76)

Le «narcissisme malin» est une variante du type psychopathique (voir aussi Kernberg, 1992: 67), et nous croyons que c'est la forme spécifique de pathologie dont était affligé L. Ron Hubbard. Si ce diagnostique est valable pour Hubbard, cela pourrait alors certainement fournir une explication à l'élaboration et à la mise en application de politiques contre des personnes perçues par lui comme des adversaires ${ }^{10}$.

\section{Dysrégulation de l'estime de soi}

Des problèmes de dysrégulation de l'estime de soi entraînent «un sentiment de supériorité et d'être unique... ; l'exagération des talents et des réalisations... [;] des fantasmes grandioses... [;] un comportement centré sur soi et autoréférentiel... [;] une attitude vantarde et prétentieuse... [; le] besoin d'attention admirative... [;] de fortes réactions face à la critique et à la défaite» (Ronningstam, 2005 : 83; voir «Diagnostic Criteria»1-4 et 9 dans American Psychiatric Association, 2000b; Lieberman, 2004: 75). Le fondateur de la Scientologie éprouvait des problèmes importants avec tous les aspects de la dysrégulation de l'estime de soi.

10. Il peut être utile de mentionner que Kernberg a indiqué que quelques personnes atteintes de narcissisme malin «peuvent présenter un comportement antisocial rationalisé - par exemple, en tant que chefs de gangs sadiques ou de groupes terroristes» (Kernberg, 1992: 78). Sans vouloir pousser les analogies aux «gangs sadiques» et aux «groupes terroristes» trop loin, à savoir qu'elles pourraient s'appliquer à la Scientologie, quelques-uns des châtiments que Hubbard a infligés à ses loyaux disciples avaient certainement des connotations sadiques. Ceux qui nous viennent immédiatement à l'esprit sont ceux de la période pendant laquelle Hubbard commandait une petite flotte de bateaux de la Scientologie, à la fin des années 1960 et au début des années 1970. Durant cette période, Hubbard a ordonné plusieurs fois comme châtiment que des personnes soient lancées pardessus bord à des hauteurs considérables (quelquefois les yeux bandés avec les mains ou les pieds attachés lâchement). Comme un détracteur de la Scientologie avait conclu au sujet de ces châtiments, «être lancé d'une telle distance [entre 4,5 et 12 mètres] dans l'eau froide de la mer, les yeux bandés et les membres attachés, devait être terrifiant» (Atack, 1990: 187). De plus, Hubbard a puni des enfants en les envoyant dans la cale aux chaînes du bateau pendant plusieurs jours (Atack, 1990: 180). Des personnes comme Paulette Cooper, qui sont devenues des cibles de la politique «fair game» de la Scientologie (comme nous l'examinerons), ont dû aussi certainement se sentir terrorisées par l'organisme. 
Aucun énoncé ne rend mieux compte du sentiment de supériorité et de la prétention fanfaronne de Hubbard qu'une série de commentaires qu'il a écrits dans une lettre à sa première femme en 1938 :

C'est peut-être fou, mais j'y crois néanmoins, j'ai de grands espoirs de pulvériser mon nom dans l'histoire si violemment qu'il deviendra légendaire même si tous les livres sont détruits. Cet objectif est le véritable objectif en autant que je suis concerné [sic.]. (Hubbard, cité dans Miller, 1987: 81)

Par conséquent, ses réalisations étaient à ses yeux grandioses et légendaires. Par exemple, dans sa première impression sur son succès en librairie de 1950, La dianétique: la science moderne de la santé mentale, il proclamait que: «La création de la dianétique constitue une étape importante pour l'Homme, comparable à la découverte du feu et supérieure à l'invention de la roue et de l'arc.» (Hubbard, 1950: ix) De même, en 1974, il a présenté son traitement réputé contre la psychose en déclarant: "J'ai réalisé une percée technique qui possiblement se classe parmi les grandes découvertes du $\mathrm{xx}^{\mathrm{e}}$ siècle. C'est certainement le plus grand progrès de 1973...» (Hubbard, 1974b, dans Hubbard, 1976d: 239)

Dans au moins deux livres qu'il a rédigés, Hubbard a plus qu'exagéré sur ses titres quand il s'est présenté comme «L. Ron Hubbard, C. E. [ingénieur civil], $\mathrm{PhD} »(H u b b a r d, 1956 a ; 1956 b)$ même s'il avait abandonné ses études collégiales et n'avait jamais obtenu son baccalauréat ni reçu de diplôme dans aucune discipline de l'ingénierie (Miller, 1987 : 57). En dépit de ces supercheries et de beaucoup d'autres au sujet de ses titres, il était complètement autoréférentiel quand il donnait des instructions à ses disciples sur la façon de procéder à l'attribution des tâches ou des postes. Dans une directive du comité de direction qu'il a écrite en 1969, il a utilisé un conférencier imaginaire pour faire comprendre son idée que si quelqu'un éprouvait des difficultés, la meilleure chose à faire alors était de «Se souvenir de la vieille maxime: Quand tout a échoué, faites Ce que Ron a dit.» (Hubbard, 1969: 260 [majuscules dans l'original]) En effet, pour une personne détenant un "poste» dans la Scientologie (selon l'appellation donnée par Hubbard) «ESSENTIELLEMENT, VOUS PORTEZ MON CHAPEAU ADMINISTRATIF POUR CE POSTE... Comme c'est réellement mon chapeau, si petit soit le poste, il doit être porté comme je le porterais.» (Hubbard, 1967b: 238 [majuscules et italique dans l'original]) 
Hubbard avait un besoin maladif d'attention et d'adulation. Comme cela a été révélé en 1984 lors d'un procès, à la fin des années 1930, Hubbard avait l'habitude de répéter ce qu'il appelait ses «affirmations», qui incluaient l'énoncé «Tous les hommes devraient être mes esclaves! Toutes les femmes devraient succomber à mes charmes! L'humanité entière devrait ramper à mes pieds et ne pas savoir pourquoi!» (Hubbard, cité dans Corydon, 1996: 58). Un exemple plus évident de narcissisme est difficile à imaginer. Nous devrions revenir aux «fortes réactions de Hubbard face à la critique et à la défaite» lorsque nous examinons la dimension maligne de son narcissisme.

\section{Dysrégulation affective}

Selon Ronningstam, «Les gens atteints de [névrose narcissique] ont à faire face à la fois à la présence d'affects forts [c'est-à-dire d'émotions], surtout la rage, la honte et l'envie, et à la faible tolérance de la nature et de l'intensité de ces sentiments.» (Ronningstam, 2005: 83) Ils ont en particulier de «forts sentiments de honte et d'envie... [,] des réactions agressives intenses aux menaces à l'estime de soi... [,] de brusques variations d'humeur... [et] des réactions intenses à la perception de l'envie des autres.» (Ronningstam, 2005: 92 : voir «Diagnostic Criteria» 8 dans l'American Psychiatric Association, 2000b; Lieberman, 2004 : $75,76,80)$ Dans le cas de Hubbard, des commentaires de Barbara Kaye (un pseudonyme) fournissent un aperçu de ce qui semble avoir été des sentiments de honte au sujet de sa mère. Elle était la secrétaire et l'amante de Hubbard en 1950 et 1951. Elle rappelle qu'alors qu'il «avait bu excessivement», il a raconté:

des histoires [g]rotesques au sujet de sa famille principalement et de sa haine envers sa mère, qui était une lesbienne et une prostituée... C'est un homme profondément malheureux. Il a affirmé que le seul être à lui avoir démontré de l'affection dans les quelques dernières années avant de faire ma rencontre était Calico, son chat. (Kaye, cité dans Miller, 1987: 169)

Il a aussi affirmé qu'il avait trouvé sa mère « au lit avec une autre femme, et qu'il était né parce que la tentative d'avortement avait échoué» (Ibid.: 168). Le souvenir de Kaye à propos de la honte de Hubbard concernant les circonstances entourant sa propre naissance a probablement un rapport avec le fait que dans son livre La dianétique, écrit en 1950, il a donné de nombreux exemples d'énergie mentale négative (ce que 
Hubbard a appelé des «engrammes») créée à la suite d'avortements échoués (Hubbard, 1950: 132-133, 156, 286).

La dysrégulation narcissique concernant l'envie «a été spécialement difficile à clarifier» (Ronningstam, 2005: 89), en partie parce que:

Les gens essaient toujours de cacher qu'ils sont envieux, ils n'expriment presque jamais leur sentiment d'une manière directe... Les expressions d'envie dans un contexte social et interpersonnel peuvent être extrêmement subtiles et dérangeantes, allant d'un comportement destructeur discret en retenant ce dont une personne a besoin..., jusqu'à briser ou détruire physiquement l'objet... (Ronningstam, 2005: 90; voir «Diagnostic Criteria» 8, dans American Psychiatric Association, 2000b)

En dépit des difficultés associées à discerner l'envie chez une personne atteinte d'un trouble narcissique, un incident qui s'est passé entre Hubbard et l'une des capitaines de son bateau, Hana Eltringham, constitue presque certainement un exemple dramatique de comportement destructeur narcissique.

Eltringham s'est jointe à la Scientologie en Afrique du Sud et en août 1967 elle est devenue membre de l'équipage de Hubbard sur son premier bateau (Miller, 1987 : 269). Elle a rapidement gravi les échelons et, finalement, Hubbard l'a nommée capitaine du navire. (Ibid.: 282) Elle travaillait souvent en étroite collaboration avec lui et elle a développé une profonde admiration pour lui. «Il était tout pour moi. Je l'aimais comme un père ou un frère, il faisait partie de ma famille. Je l'aimais réellement beaucoup [.] J'aurais fait n'importe quoi pour lui...» (Eltringham, cité dans Miller, 1987: 287) Sur le plan sentimental, cependant, elle était aussi "profondément en amour» avec un autre membre, John O'Keefe, «et l'avait été depuis quelque temps» (Corydon, 1996: 65). Essentiellement, elle éprouvait un attachement émotionnel profond pour quelqu'un d'autre qu'Hubbard (qui était au courant de la relation entre Eltringham et O'Keefe [Ibid.: 68]), et - d'une manière cohérente avec la destruction narcissique envieuse - il s'était donné pour but de la détruire.

Hubbard a nommé O'Keefe capitaine de l'un de ses bateaux et lui a donné des ordres d'appareillage - dont le contenu allait devenir un sujet de dispute. O'Keefe a suivi les instructions et, par la suite, il a insisté pour dire que Hubbard les lui avait données, mais elles ont conduit le bateau directement dans un violent ouragan qui a failli le détruire. O'Keefe a survécu à l'épreuve, au moyen de grands efforts cependant, mais le bateau n'était pas accosté au port où Hubbard s'attendait à ce 
qu'il le soit. Furieux, Hubbard a créé un «comité de la preuve» (c'est-àdire, un tribunal interne de la Scientologie) ${ }^{11}$ pour décider de l'aptitude d'O'Keefe, de sa compétence et de sa loyauté, ne faisant alors preuve d'aucune considération pour l'épreuve qu'il avait traversée. Eltringham se trouvait être à côté de Hubbard quand un de ses messagers lui a remis une instruction de Hubbard lui-même l'informant qu'elle allait présider le comité de la preuve chargé de délibérer au sujet de son amant. «Il s'est retourné et, la face ornée d'un demi-sourire, il a dit: "Justice poétique", n'est-ce pas?» (Eltringham citant Hubbard dans Corydon, 1996: 68)

De la façon dont nous interprétons cette action, Hubbard enviait l'autre relation émotionnelle forte d'Eltringham et il l'a brisée en plaçant délibérément Eltringham dans une position où elle aurait à juger son amant. Comme elle l'a indiqué, le pouvoir absolu que Hubbard détenait sur l'organisation signifiait que: "Je savais que je devais trouver John coupable. Absolument! Il n'y avait aucune autre issue...» (Eltringham, cité dans Corydon, 1996: 69) La stratégie a fonctionné: le comité qu'Eltringham présidait a reconnu O'Keefe coupable, même si en son for intérieur elle pensait qu'il était innocent. Par conséquent, O'Keefe a quitté la Scientologie et Eltringham, tandis qu'elle est demeurée une admiratrice loyale de Hubbard pendant plusieurs autres années. Hubbard avait réussi à détruire leur relation. Ce n'est que plus tard qu'Eltringham a réalisé que Hubbard était probablement dans l'erreur quand il a donné l'ordre d'appareillage à O'Keefe.

Des gens comme Eltringham qui ont travaillé en étroite collaboration avec Hubbard ont vu (et ont subi) ses remarquables variations d'humeur (Miller, 1987 : 269-270; Corydon, 1996: 104). Ronningstam a précisé que ces «variations d'humeur brusques» chez les gens atteints de névrose narcissique comprenaient des éléments comme de «brèves dépressions réactionnelles», de l'hypomanie ${ }^{12}$, «l'abus périodique de

11. Bien que pour des personnes n'appartenant pas à la Scientologie, un comité de la preuve ressemble à une audience ou à un tribunal, Hubbard insistait pour expliquer qu'un «comité de la preuve» n'est pas une cour. C'est simplement une commission avec des pouvoirs juridiques chargée de trouver les faits...» (Hubbard, 1976a: 91 [caractères gras dans l'original]). Il a aussi dit que c'était «la plus grave forme d'action morale» (Hubbard, 1976a: 91). «Les pouvoirs juridiques» que le comité possédait ne valaient, bien entendu, que dans les limites du système de «justice» interne de la Scientologie.

12. Les épisodes hypomaniaques entraînent une élévation de l'humeur pendant au moins quatre jours, accompagnée d'au moins trois des éléments suivants: de la grandiosité, une réduction du sommeil, de la verbomanie, un flot continu de pensées, de «l'inattention» ou une «augmentation des activités orientées vers un but» (American Psychiatric Association, 2000a). 
drogues», de l'hypochondrie et des "préoccupations au sujet du corps» (Ronningstam, 2005: 91). Ces traits décrivaient Hubbard presque parfaitement. Au début des années 1950, ses variations d'humeur étaient parfois si importantes que Barbara Kaye était convaincue qu’«il était atteint de trouble bipolaire avec des tendances paranoïaques» (Miller, 1987: 175). Eltringham a eu par la suite la réflexion suivante au sujet de son tempérament explosif: «les hurlements acharnés - juste une indignation surprenante qui sortait de lui quand quelque chose n'allait pas bien» Atack, 1990: 250-251; Eltringham, cité dans Corydon, 1996: 64, voir 65, 68-69, 74).

Des problèmes liés à l'hypocondrie et aux préoccupations au sujet du corps sont notés dans le dossier médical de Hubbard immédiatement après la Deuxième Guerre mondiale. Le biographe Russell Miller a déniché ceci:

Hubbard a été un patient du Oak Knoll Naval Hospital pendant trois mois après la guerre, bien que les médecins n'arrivaient pas à trouver ce qui précisément n'allait pas avec lui. Il n'était certainement ni aveugle ni impotent, mais il semblait souffrir de maux et de peines mineures inguérissables. Son dossier médical indique qu'il a été examiné de façon exhaustive, presque chaque semaine, se plaignant de maux de tête, de rhumatisme, de conjonctivite, de douleurs au côté, de maux d'estomac, de douleurs à l'épaule, d'arthrite, d'hémorroïdes... il semblait ne pas y avoir de limites à ses souffrances. Parfois, les médecins pouvaient trouver des symptômes, parfois ils ne le pouvaient pas. (Miller, 1987: 112) ${ }^{13}$

Il semble plausible qu'au moins quelques-unes de ces supposées affections - ou encore plus plausible que plusieurs d'entre elles - avaient très peu à voir avec le corps, sinon pas du tout (voir Corydon, 1996: 309).

À une autre étape de sa vie, Hubbard semblait avoir la phobie des microbes et des senteurs. Une jeune femme, Tonya Burden, a émis des observations importantes sur ces phobies dans une déclaration sous serment en 1980. Au début des années 1970, en tant que membre (alors âgée de 13 ans) du personnel de gestion de Hubbard, la Sea Organization (ou Sea Org), Burden a d'abord dû se soumettre à un programme de

13. Voyez par exemple l'«examen orthopédique spécial» de Hubbard, mené le 01-0851 (sans doute le $1^{\text {er }}$ août 1951) par C. L. Williams, M. D., examen durant lequel on n'a pu trouver les présumées maladies, «la bursite avec calcification», «l'arthrite multiple» ou «l'ulcère duodénal», maladies que Hubbard prétendait avoir. 
«formation et travail» pour adolescents appelé l'Estates Project Force (EPF). Une des tâches pénibles du programme consistait à nettoyer des vêtements six heures par jour. "Les vêtements étaient frottés à la main dans une chaudière, et j'ai reçu la directive de rincer chaque article dans 13 chaudières différentes.» Elle a ajouté par la suite que Hubbard «explosait fréquemment s'il trouvait de la poussière ou de la saleté, ou si les vêtements sentaient le savon. C'est la raison pour laquelle nous utilisions treize chaudières pour le rinçage» (Burden, 1980:4, 6 [souligné dans l'original]). Hubbard, semble-t-il avait peur de la saleté et était dégoûté par les odeurs.

Le trait narcissique de la dysrégulation affective le plus frappant chez Hubbard était cependant ses «réactions agressives intenses aux menaces à l'estime de soi» (Ronningstam, 2005 : 92; Phillips et al., 2008). Ces réactions étaient si dramatiques que nous les examinerons dans la partie de notre analyse portant sur les aspects malins de la névrose narcissique de Hubbard.

\section{Dysrégulation des relations interpersonnelles}

Toujours selon les recherches actuelles sur la névrose narcissique, Ronningstam a indiqué que: «Les individus narcissiques sont habituellement identifiés par leur modèle interpersonnel précis, qui se caractérise plus ou moins ouvertement par une attitude arrogante et hautaine et par un comportement contrôlant et qui s'accorde des droits.» (Ronningstam, 2005: 99; voir «Diagnostic Criteria» 5 et 9 dans American Psychiatric Association, 2000b; Kernberg, 1975: 228) Élaborant ensuite, elle a précisé que ces traits incluent «un comportement arrogant et hautain..., s'accorder des droits... [, une] capacité d'empathie réduite..., le contrôle et l'hostilité interpersonnels... [et] un manque d'engagement soutenu envers les autres» (Ronningstam, 2005 : 99-100; voir «Diagnostic Criteria» 5, 6 et 7 dans American Psychiatric Association, 2000b). Quant aux autres traits narcissiques, Hubbard en a fait la démonstration, souvent de façon très dramatique.

Concernant ses supposées réalisations, Hubbard croyait qu'elles étaient dignes des plus grands éloges. Essentiellement il avait le sentiment qu'il méritait un Prix Nobel «pour sa découverte ou sa création Purification Rundown», selon Armstrong (1984a: 1503; voir Atack, 1990 : 260). C'était un programme qui supposément pouvait purger le 
corps des drogues et des résidus de la radiation. À la fin de 1979, Hubbard avait affecté «des fonds illimités» à son projet, qui en fin de compte a échoué (Armstrong, 1984b: 1790).

Bien qu'il ait eu le sentiment très fort qu'il avait droit aux honneurs pour ses propres projets, il méprisait avec arrogance les personnes qu'il percevait comme ennemies et qui s'opposaient à lui, surtout les psychiatres. En 1982, il a écrit au sujet de «ceux qui prétendent gérer le monde et ont en fait un intérêt direct (pour leurs propres aspirations et profits)» et qui, prétendait-il, «ont organisé des attaques de grande envergure» contre son organisation (Hubbard, 1982: 1):

Honnêtement, mes amis, une revue de ces batailles au cours des trente-deux dernières années ne peut que provoquer un rire méprisant. L'ennemi, perché dans son arbre ou se balançant par la queue, a été à peu près aussi efficace qu'un de ces singes psychologues épluchant la matraque d'un policier en pensant que c'est une banane et ensuite la lançant pour seulement atteindre le chef grand singe à la figure. (Ibid.: 2)

En amenant son personnel à entreprendre le projet Prix Nobel et d'autres projets, il a essayé de maintenir un contrôle serré sur les projets ainsi que sur les gens qui travaillaient pour lui, et il leur donnait souvent des ordres d'une manière extrêmement hostile. Par exemple, pendant qu'il était sur le bateau Apollo, au début des années 1970, Hubbard a créé l'Organisation des messagers du Commodore (OMC) et il a engagé pour personnel des adolescentes qui «s'habillaient dans de mignons petits uniformes bleu foncé avec cordons or» (Miller, 1987: 301).

[Les filles de l'OMC] étaient formées pour transmettre les ordres de Hubbard en utilisant les mêmes mots et le même ton de voix: s'il était dans une crise de colère et qu'il hurlait, le messager s'en allait à toute vitesse et transmettait le même message au coupable. Personne n'osait contester quoi ce soit de ce que le messager avait dit; personne n'osait désobéir à ses ordres. (Miller, 1987: 301-302)

En plus de relayer les ordres à l'équipage, une autre fonction des filles de l'OMC consistait à servir Hubbard - le servir au doigt et à l'œil. Une ancienne messagère, par exemple, a confirmé dans une déclaration sous serment:

En tant que domestique, j'étais assise à l'extérieur de sa chambre, et dès qu'il prononçait le mot «messenger», je l'aidais à sortir du lit. Je répondais donc en l'aidant à sortir du lit, j'allumais sa cigarette, faisais couler la douche, préparais ses articles de toilette et l'aidais à s'habiller. Ensuite, je 
courais vers son bureau pour vérifier, en espérant qu'il passe l'inspection gant blanc [c'est-à-dire sans aucune poussière]. (Burden 1980: 6)

Ces interactions avec les filles de l'OMC démontrent le sentiment très prononcé de Hubbard d'avoir des droits. La façon de les utiliser pour crier des ordres à leurs camarades de bord suggère les difficultés qu'il avait dans ses relations interpersonnelles.

Ces difficultés sont encore plus évidentes dans ses interactions avec sa famille. Il ne pouvait faire preuve d'empathie envers aucun de ses membres. En novembre 1976, il apprend que son fils Quentin s'est suicidé. Une fille de l'OMC a par la suite rapporté avoir entendu Hubbard «crier le plus fort qu'il pouvait: "Cet espèce de salaud de fils! Cet espèce de salaud de fils! Regarde ce qu'il m'a fait! Espèce de salaud...” (cité dans Miller, 1987: 344) ${ }^{14}$. Comme on pourrait s'y attendre, après avoir été mis au courant de cet incident, Hubbard n'avait pas la capacité de maintenir des engagements interpersonnels soutenus, même avec de nombreux membres de sa famille. Il s'est marié trois fois (dont un mariage bigame); son fils Quentin s'est suicidé; son fils le plus âgé a dénoncé son père et a passé des années à parler contre lui (parfois il se rétractait [Corydon, 1996: 11-13, 55-58]), et sa fille Alexis, dont il vivait séparé, a refusé de signer «une déclaration bizarre affirmant que L. Ron Hubbard JUNIOR [était] son père réel» (Corydon, 1996: 13 [majuscules dans l'original]). En 1984, un juge a écrit au sujet de la troisième femme de Hubbard, Mary Sue, qu'elle «semblait certainement être une personne misérable. Elle a été forcée de démissionner de son poste de contrôleur [du Guardian's Office], condamnée et emprisonnée pour des actes criminels [pour son rôle dans les opérations illégales de la Scientologie contre le gouvernement des États-Unis, dans les années 1970] et abandonnée par son mari.» (Juge Brekenridge, cité dans Corydon, 1996: 255) En plus d'un dysfonctionnement familial tragique, de nombreuses personnes qui à différents moments ont travaillé en étroite collaboration avec Hubbard (telles que Laurel Sullivan, Hana Eltringham, John McMaster, Richard DeMille, etc.) ont coupé leurs

14. Kernberg constate, par exemple, que les gens atteints de névrose narcissique «sont en particulier incapables d'éprouver des sentiments sincères de tristesse et de nostalgie; leur incapacité à ressentir des réactions dépressives est une caractéristique de base de leur personnalité. Lorsqu'ils sont abandonnés ou déçus par des personnes, ils peuvent montrer ce qui semble à la surface des signes de dépression, mais après un examen plus approfondi, ce qui émerge est de la colère et du ressentiment, chargés de désirs vengeurs, plutôt que de la tristesse réelle pour la perte d'un être qu'ils appréciaient» (Kernberg, 1975: 229). 
liens avec lui et ont parlé publiquement de mauvais traitements (voir, par exemple, Miller, 1987 : 214-215 ; Corydon, 1996: 37-40, 206-207, 350-352). Ses traits narcissiques pathologiques ont apparemment anéanti sa capacité à entretenir des relations sérieuses avec les autres pendant de longues périodes.

\section{Narcissisme malin}

Pour l'expliquer simplement, les personnes qui font du mal aux autres quand elles expriment les traits narcissiques de leur personnalité sont atteintes de narcissisme malin, selon Kernberg $(1975,1992,1998)$. Cette forme de narcissisme se caractérisée par «un comportement antisocial déclaré, passif ou actif, par des traits paranoïaques, de l'agressivité conforme au moi et du sadisme qui peuvent être dirigés envers les autres aussi bien qu'envers soi-même» (Ronningstam, 2005 : 106). Quelques recherches suggèrent «que le narcissisme malin peut s'exprimer par une violence apparemment autojustifiable, une cruauté sadique ou de l'autodestruction dans lesquelles l'agressivité et le sadisme sont combinés avec la joie et l'estime de soi accrue» (Ronningstam, 2005 : 106). Dans les cas extrêmes, les personnes atteintes de narcissisme malin peuvent devenir des meurtriers qui considèrent que «tuer est un acte de vengeance justifié, un effort désespéré pour obtenir le contrôle et pour protéger et augmenter l'estime de soi» (Ronningstam, 2005 : 107).

Parce que de nombreux traits du narcissisme malin se manifestent dans les relations sociales, nous avions reporté l'examen de certains traits narcissiques jusqu'à maintenant. Nous revenons particulièrement à présent sur un trait concernant la régulation de l'estime de soi (c'està-dire «de fortes réactions face à la critique et à la défaite » [Ronningstam, 2005 : 83]) et sur une caractéristique de la régulation affective (c'est-àdire «des réactions agressives intenses aux menaces à l'estime de soi» [Ronningstam, 2005: 92]). Il nous semble que ces traits généraux du caractère vont probablement se manifester de façon violente dans la vie des personnes atteintes de narcissisme malin (Kernberg, 1984: 257; 1992: 67), comme ils se sont manifestés dans la vie de L. Ron Hubbard. Une caractéristique qui attire cependant notre attention au sujet de Hubbard est qu'il avait une organisation entière de disciples - des dizaines de milliers au moins - sous ses ordres, y compris un noyau dévoué de membres à temps plein de la Sea Org, qui étaient préparés à 
agir sous ses ordres et désireux de le faire. Nous soutenons que Hubbard a clairement démontré qu'il était atteint de narcissisme malin dans les différentes doctrines organisationnelles qu'il a élaborées et dans les structures et les politiques créées pour les mettre en application ${ }^{15}$.

\section{La formation de l'0ffice du gardien}

À partir de la publication de La dianétique: la science moderne de la santé mentale (1950) jusqu'à sa mort, en 1986, l'évolution de la doctrine de Hubbard a suscité des critiques considérables de la part de nombreuses organisations. Dans les médias, les premières critiques du livre (souvent faites par des psychiatres et des médecins d'autres spécialités) ont dénoncé la dianétique comme inefficace et comme une dangereuse substitution aux traitements psychiatriques réguliers (Atack, 1990: 160-161). Par exemple, peu après la publication de La dianétique, un ancien président de l'American Psychiatric Association et psychiatre de renom, William Menninger, concluait: «Cela peut possiblement faire beaucoup de mal. Il est évident que [Hubbard] a simplifié à l'excès la personnalité humaine, à la fois dans sa structure et son fonctionnement, et mon impression est que les résultats contiennent des affirmations démesurées et très exagérées.» (cité dans Clarke, 9 septembre 1950: 3) Un psychiatre de Beverley Hills, en Californie, Frederick J. Hacker, a également discrédité la création de Hubbard en affirmant: «Si ce n'est pas de notre sympathie pour la souffrance morale des personnes perturbées, la science [sic] ainsi appelée la dianétique pourrait être rejetée pour ce qu'elle est - un projet astucieux pour plonger avec impunité dans les poches de personnes crédules» (cité dans Clarke, 9 septembre 1950 : 3, 16). Bien qu'au début le mouvement de la dianétique ait été populaire, l'intérêt a rapidement diminué, à cause des discussions dans le public au sujet de la valeur et de l'efficacité de ses pratiques (voir Wallis, 1977: 87).

15. Il vaut la peine de mentionner ici une observation de Kernberg: «Quand des personnes narcissiques se trouvent dans une situation importante - par exemple, à la tête d'une institution politique ou d'un groupe social - elles aiment s'entourer d'admirateurs auxquels elles s'intéressent au début, tant que c'est nouveau. Quand elles ont le sentiment qu'elles ont obtenu toute l'admiration dont elles avaient besoin, elles perçoivent leurs admirateurs comme des "ombres" une fois de plus et elles les exploitent et les maltraitent sans pitié. En même temps, ces [personnes narcissiques] sont extrêmement offensées quand un de leurs "esclaves" veut devenir libre» (Kernberg, 1975: 236). 
Après l'expansion de la théorie de la dianétique à la suite de la création de la Scientologie en $1952^{16}$, Hubbard a fait face à des critiques continues sur un certain nombre de fronts. Les psychiatres ont repris leur dénigrement de l'idéologie de Hubbard (pour des exemples, voir Atack, 1990: 219, 392), et son organisation a subi des attaques de gouvernements partout dans le monde (pour des exemples, voir Miller, 1987: 247, 252-253; Atack, 1990: 142). En tant que créateur, unique théologien et leader institutionnel de la Scientologie, Hubbard a considéré les attaques contre son idéologie et son organisation comme des attaques personnelles. Comme il l'a révélé dans une publication confidentielle sur la justice: «Les gens attaquent la Scientologie; je n'oublie jamais [et] j'y pense toujours, et je finis toujours par régler mes comptes.» (Hubbard, 1959: 1)

En faisant la démonstration que le trouble de la personnalité paranoïaque associé au narcissisme malin accompagne des «expériences d'échec important, de trahison ou d'humiliation» (Ronningstam, 2005: 131), Hubbard a réagi aux critiques externes en prétendant qu'il était victime d'une conspiration internationale. Il a affirmé que la Fédération mondiale pour la santé mentale et des membres d'associations nationales équivalentes étaient bien établis dans des agences privées et gouvernementales qui étaient opposées à la Scientologie (Hubbard, 1968e; LRH Aides, 1969: 3-7). Bien que Hubbard ait fait des allusions répétées à la conspiration des psychiatres contre lui durant le temps qu'il a été le chef de la Scientologie, il n'a jamais étayé ses affirmations sur des preuves.

En août 1965, une commission d'enquête sur la Scientologie s'est réunie en Australie pour investiguer sur des «allégations de chantage et d'extorsion et sur des accusations voulant que [cela] affectait le "bienêtre mental” des étudiants de l'Université de Melbourne» (Miller, 1987: 25). Comme le public est devenu plus suspicieux, Hubbard a répondu en formulant des politiques qui reflétaient de la dysrégulation affective et de l'estime de soi exagérée, qui sont des traits du narcissisme malin.

Faisant la démonstration à la fois de ses «fortes réactions devant la critique et à la défaite» (Ronningstam, 2005: 83) et de ses «réactions agressives intenses devant des menaces envers son estime de soi» (Ibid.:

16. La première conférence de Hubbard sur la Scientologie a eu lieu le 3 mars 1952 (Hubbard, 1976b: 218), bien qu'il ait attiré l'attention des lecteurs dans une publication de décembre 1951 sur le fait que la nouvelle science de la Scientologie serait bientôt présentée (Hubbard, 1951: 41). 
92), Hubbard est devenu préoccupé par ces critiques externes, au point de rédiger des lettres de politiques sur l'identification de soi-disant ennemis. Ces politiques concernaient ce qu'il appelait une «personne suppressive» ou un "groupe suppressif» qui «cherchait activement à léser ou à abolir la Scientologie ou à s'en prendre à un scientologue par des "actions répressives"» (Hubbard, 1965a: 1). Il a décrit les "actions répressives» comme des «actions ou des omissions entreprises délibérément pour abolir, réduire ou nuire à la Scientologie ou aux scientologues» (Hubbard, 1965a: 553). Dans une lettre de politiques supplémentaire, Hubbard a fourni un commentaire complet sur les caractéristiques, les motifs et les actions des "personnes supressives» (Hubbard, 1965b). La fixation de Hubbard sur les critiques est également le reflet de «son extrême sensibilité et de sa tendance à surinterpréter les rejets ou les déceptions» (Malmquist, 1996: 169), signes également associés au narcissisme malin.

Peu après que Hubbard a commencé à intégrer son discours sur les ennemis de la Scientologie aux politiques de l'organisation, la Scientologie a connu un échec remarquable quand la commission d'enquête sur la Scientologie en Australie a publié son rapport sur l'organisation. Après la sortie du rapport, «l'État de Victoria a adopté le Psychological Practices Act, qui effectivement déclarait illégale la Scientologie et autorisait le procureur général à saisir et à détruire tous les documents et tous les enregistrements de la Scientologie» (Miller, 1987: 254). Durant l'année suivante, la Grande-Bretagne (pays où vivait Hubbard) a aussi entrepris une enquête sur la Scientologie.

Puisque la surveillance internationale de la Scientologie augmentait, Hubbard a réagi en lançant la «Public Investigation Section», une division de l'organisation ayant pour but d'accumuler des renseignements et d'enquêter sur les détracteurs (Atack, 1990 : 160). Durant le mois suivant, il a changé le nom de la Public Investigation Section pour celui de l'Office du gardien (GO pour Guardian's Office) (Atack, 1990: 161) et il a annoncé que sa femme, Mary Sue, superviserait la division.

La principale mission de l'Office du Gardien était «d'aider LRH [Hubbard] à formuler et à faire respecter des politiques, de protéger la Scientology Orgs, les scientologues et la Scientologie, et de faire de la promotion à long terme» (Hubbard, 1966b: 1). Le GO a adhéré aux politiques de Hubbard, mais celui-ci ne permettait aux autres membres du personnel du GO d'en élaborer de nouvelles que si elles étaient consistantes avec celles en place, qui elles, bien entendu, avaient déjà 
été formulées par lui (Hubbard, 1966b: 1). Le refus de Hubbard de renoncer au contrôle absolu de l'idéologie de la Scientologie et des directives de l'organisation révèle la présence de problèmes de régulation de l'estime de soi associés au narcissisme malin, tout comme le sentiment d'être supérieur et d'être unique, et un comportement centré sur soi et autoréférentiel (Ronningstam, 2005: 83).

Afin de maintenir davantage l'autorité de Hubbard et de faire respecter ses directives, le GO opérait sur une base internationale. Son siège social était situé en Grande-Bretagne, et il y avait huit bureaux satellites dans le monde. Chaque GO comprenait six bureaux qui exécutaient les politiques de Hubbard: les bureaux de l'information (nommé au début le bureau des renseignements), des relations publiques, du contentieux, des finances et de la coordination sociale. Le bureau de l'information et celui du service du contentieux collaboraient pour déterminer qui étaient les détracteurs de la Scientologie et s'occuper d'eux.

Le bureau de l'information était, en quelque sorte, l'agence de renseignements de la Scientologie. La division rassemblait de l'information à l'interne et à l'externe dans le but d'identifier les détracteurs et d'accumuler des données sur eux. Le bureau comprenait aussi la section des opérations, qui préparait et exécutait les campagnes contre les ennemis de la Scientologie (Atack, 1990: 219). Le bureau du contentieux s'occupait des affaires juridiques de la Scientologie; dans ses opérations, il intentait des poursuites contre les ennemis, invariablement avec l'accord de Hubbard:

Le but du procès est de harceler et de décourager plutôt que de gagner. La loi peut être facilement utilisée pour harceler, et une certaine quantité de harcèlement contre quelqu'un, qui simplement se trouve dans une situation fragile de toute façon, sachant bien qu'il n'est pas autorisé, suffira généralement pour l'anéantir professionnellement. Si cela est possible, bien entendu, ruinez-le complètement. (Hubbard, 1955 : 157)

Durant tout le temps que le GO a été en fonction, le bureau du contentieux a abondamment utilisé le système juridique pour intimider et harceler les soi-disant adversaires de la Scientologie (Kumar, 1997).

Ensemble, les bureaux de l'information et du contentieux ont été des divisions permanentes de l'organisation, mandatées pour déterminer qui étaient les ennemis de la Scientologie et exécuter des campagnes de harcèlement planifiées et à long terme dans le but de les faire taire. L'existence de structures dans l'organisation autorisant à identifier et à 
attaquer les ennemis selon les directives de Hubbard est une indication des «réactions agressives intenses aux menaces à l'estime de soi» (Ronningstam, 2005: 92) et du «comportement agressif, sadique, vengeur» de la personnalité narcissique (Ibid.: 110). La création et les opérations des bureaux reflètent aussi la propension des personnes atteintes de narcissisme malin à «mettre en œuvre des plans pour anéantir une autre personne, souvent celle qui se trouve sur leur chemin pour les empêcher d'obtenir encore plus de gloire» (Malmquist, 1996: 165).

\section{Les politiques de l'0ffice du gardien pour s'occuper des ennemis}

Dans le but d'exécuter les directives de Hubbard pour faire taire les détracteurs de la Scientologie, le GO a observé plusieurs politiques clés décrivant comment s'occuper des ennemis. Les politiques sont également le reflet du narcissisme malin de Hubbard, en ce qu'elles sont des «réactions agressives» aux menaces extérieures (Ronningstam, 2005 : 92) et nous informent des opérations continuelles contre les détracteurs. Il a exposé le principe directeur déterminant le traitement des ennemis de la Scientologie dans la politique «Fair Game». (Dans la politique «Penalties for Lower Conditions» (Hubbard, 1967a), Hubbard a affirmé que les personnes déclarées «suppressives» devaient être traitées selon la politique «Fair Game» et que «n'importe quel scientologue [p]ourrait leur enlever leurs biens ou les blesser par quelque moyen sans encourir aucune mesure disciplinaire... [et] elles [p]ourraient être escroquées, poursuivies ou anéanties, ou on pourrait leur mentir» (Hubbard, 1967a).

La politique «Fair Game» a reçu un accueil hostile, et Hubbard a émis une autre politique à la suite de la réaction violente du public. Dans "Cancellation of Fair Game», il a affirmé que «[1]a pratique de discréditer "Fair Game" allait cesser» (Hubbard, 1968a). Dans le même document de politiques, il a cependant continué en soutenant que «[c]ette PL [lettre de politiques] n'annule aucune politique sur le traitement et la gestion d'une SP [personne suppressive, SP pour Suppressive Person]» (Hubbard, 1968a). L'annulation de la politique par Hubbard avait évidemment pour but de calmer la critique, quoiqu'en fait, les pratiques décrites dans la politique «Fair Game» allaient être poursuivies. 
Lorsque le bureau des renseignements identifie des ennemis grâce à la collecte d'informations, ils deviennent des «personnes suppressives» et soumises aux aléas découlant du fait d'avoir été déclarées comme devant être traitées selon la politique «Fair Game». Cette politique fait la démonstration que Hubbard souffrait de rage narcissique et des problèmes qui y sont associés, «le besoin de vengeance, de redresser un tort, de réparer une blessure par quelque moyen que ce soit et une compulsion profondément enracinée et acharnée dans la poursuite de ces buts...» (Kohut, 1972: 380; voir Ronningstam, 2005: 86).

Pendant que la politique «Fair Game» était en vigueur, le GO a organisé des campagnes contre des «personnes suppressives» dans le but de satisfaire les besoins de rage narcissique de Hubbard. Comme la politique le précisait clairement, les «personnes suppressives» pouvaient être «blessées par n'importe quel moyen» (Hubbard, 1967a: 1), et les membres du GO répondaient avec ferveur aux ordres de Hubbard. Les documents du GO ont démontré que certaines activités illégales constituaient des tactiques acceptables que des agents de la Scientologie ont utilisées contre les détracteurs et les personnes considérées comme ennemies. Des opérations illégales et secrètes ont en effet été menées par le GO contre le gouvernement des États-Unis au milieu des années 1970 et ont conduit à la condamnation et à l'emprisonnement de onze scientologues haut placés. En prononçant la peine contre neuf des onze agents du GO, le juge qui présidait le procès a résumé:

[...] la nature radicale et incroyable de la conduite criminelle des défendeurs et de l'organisation qu'ils dirigeaient. Ces crimes incluent l'infiltration et le vol de documents à un certain nombre d'organisations privées nationales et mondiales très en vue, à des cabinets d'avocats et à des journaux; la mise en œuvre de campagnes de salissage et de poursuites sans fondement pour anéantir des individus qui avaient voulu exercer leur droit à la liberté d'expression selon le premier amendement; faire porter les soupçons sur des citoyens qui avaient critiqué la Scientologie, y compris par la contrefaçon de documents, ce qui a conduit à la mise en accusation d'au moins une personne innocente; [et] la violation des droits civils de citoyens et de fonctionnaires très en vue. (United States District Court For the District of Columbia, 1979a: 2-3)

Hubbard était un coconspirateur non inculpé pour ces crimes (United States District Court For the District of Columbia, 1979b: 69), puisque «L. Ron Hubbard, en vertu de son rôle de fondateur et de chef de la Scientologie, supervisait dans l'ensemble l'Office du gardien» (United 
States District Court For the District of Columbia, 1979a: 7). La mention du juge de «la contrefaçon de documents qui a conduit à la mise en accusation d'au moins une personne innocente» fait allusion à la campagne du GO contre une journaliste de New York, Paulette Cooper, et les actions contre elle démontrent de façon dramatique comment la Scientologie met en pratique des politiques qui reflètent le narcissisme malin de Hubbard.

\section{«Operation Freakout » contre Paulette Cooper}

En 1969, Paulette Cooper a publié un article intitulé «The Tragi-Farce of Scientology", qu'elle a inclus dans son livre The Scandal of Scientology, publié en 1971. Les scientologues ont riposté en montant une opération « Fair Game» contre elle. Les tactiques incluaient quatre poursuites, entre autres, pour menaces d'attentat à la bombe, qui ont conduit à sa mise en accusation (City of Clearwater, 1982; voir New York Times, 22 janvier 1979). Paulette Cooper a reçu ses premières menaces de mort dans le mois au cours duquel son premier article sur la Scientologie a été publié et, par la suite, elle a découvert des preuves que son téléphone était sur écoute (City of Clearwater, 1982: 8; voir New York Times, 22 janvier 1979). Ses voisins ont reçu des lettres qui mentionnaient qu'on essaierait de la briser; elle a commencé à recevoir des appels téléphoniques «très, très inquiétants»; ses parents ont été harcelés, et un inconnu a commencé à lui poster des copies de pages d'un journal intime qu'elle avait écrit dans sa jeunesse (City of Clearwater, 1982: 9, 11, 18). Quand Cooper a réagi en devenant la première personne à intenter une poursuite contre la Scientologie, l'organisation a intensifié sa campagne contre elle. La campagne de la Scientologie, qui était prolongée et agressive en riposte aux publications et à la poursuite de Cooper, démontre l'étendue de la «rage narcissique» et du «besoin de vengeance» de Hubbard (Kohut, 1972: 380). En appliquant de nombreuses méthodes destinées à la harceler et à l'intimider pour la faire taire, les membres du GO faisaient précisément la preuve du narcissisme malin de Hubbard.

Plusieurs instructions détaillées ont exposé comment les scientologues allaient mettre en application la politique "Fair Game», et ses agents semblent avoir suivi toutes les stratégies recommandées dans leurs opérations contre Cooper. Hubbard a recommandé des «enquêtes 
bruyantes» comme méthode efficace pour provoquer la méfiance envers les détracteurs et la perturbation de leur vie personnelle et professionnelle (Hubbard, 1966a). Les scientologues qui exécutaient de telles enquêtes devaient communiquer avec tous les collègues de l'entreprise d'un ennemi et les informer que la personne faisait l'objet d'une enquête pour violation des libertés religieuses (Atack, 1990: 167-168). Le but de la tactique consistait en fin de compte davantage à propager la méfiance envers les ennemis qu'à servir de méthode d'enquête.

Quand Cooper a poursuivi la Scientologie pour harcèlement, des agents de l'organisation ont inclus une «enquête bruyante» dans leurs activités, afin de la faire taire. Cooper a affirmé que, durant leur enquête, les scientologues ont déformé la vérité dans leur tentative de l'intimider et de la faire taire (Supreme Court of the State of New York, 1972 : 1-5). Les mensonges comprenaient «une terrible lettre diffamatoire [qui] a été postée à tous mes voisins. Elle ne contenait rien de vrai» (Cooper, cité dans New York Times, 22 janvier 1979: A-14), en plus des fausses allégations voulant que «Sa langue soit sensiblement enflée à cause d'une maladie transmise sexuellement contractée par le passé» (Ibid.) ${ }^{17}$.

Les «Noisy Investigations» sur Cooper et les autres soi-disant ennemis concrétisaient le désir de Hubbard de discréditer, de harceler et de perturber ses détracteurs, comme le faisait aussi la politique «Dead Agenting» (Hubbard, 1966a). Dans une lettre de politiques disponible au public, Hubbard a affirmé: «La technique pour prouver que des paroles sont fausses est appelée DEAD AGENTING. Quand l'agent ennemi fournit de fausses informations, ceux qui croyaient en lui savent maintenant qu'il n'est pas digne de confiance et veulent le tuer - ou au moins cessent de croire en lui» (Hubbard, 1972: 422 [majuscules dans l'original]). De la façon dont cela nous est présenté, la politique semble faire davantage que corriger des mensonges, elle conduit à discréditer les personnes qui les ont propagés. Dans une lettre de politiques «confidentielle» au sujet de «Dead Agenting» dix-huit mois plus tard, Hubbard a cependant affirmé que «Dead Agenting» impliquait «fournir à la presse la preuve même du crime sexuel épouvantable, sanguin des agresseurs»

17. Une copie de cette lettre diffamatoire se trouve dans les dossiers de la Stephen A. Kent Collection of Alternative Religions, à la bibliothèque de l'Université de l'Alberta. Elle ne contient qu'une seule page non datée, toute dactylographiée en lettres majuscules avec comme objet: d'«Un voisin concerné» à «Cher ami locataire». 
(Hubbard, 1974a: 4). En attaquant publiquement ses ennemis, Hubbard utilise tous les moyens à sa disposition pour discréditer la légitimité de ses détracteurs et réduire, sinon éliminer, l'influence de leurs critiques sur lui et son groupe. Les politiques et les tactiques de la Scientologie représentent, par conséquent, la concrétisation de la rage narcissique de Hubbard et «les plans ultérieurs pour anéantir une autre personne, souvent celle qu'il voit se dresser sur son chemin pour l'empêcher d'obtenir encore plus de gloire» (Malmquist, 1996: 165).

Après que Cooper eut intensifié le conflit en poursuivant l'organisation pour harcèlement, la Scientologie a appliqué sa politique «Dead Agenting» contre elle. Cooper a affirmé, par exemple, que la Scientologie avait publié des mensonges à son sujet dans les médias dans une tentative pour ruiner sa carrière en détruisant sa crédibilité en tant qu'écrivaine (Supreme Court of the State of New York, 1972: 6). Aux alentours de 1974, le ministre des Relations publiques des États-Unis de l'Église de Scientologie en Californie a fait circuler ce qu'il appelait un «False Report Correction» au sujet de son livre Scandal of Scientology, alléguant (parfois avec raison, souvent à tort) de nombreuses erreurs factuelles ${ }^{18}$. Le rapport contenait aussi une copie d'une déclaration qu'un avocat des éditeurs britanniques de l'article de Cooper «Tragi-Farce of Scientology» a lue en cour, dans laquelle elle s'excusait et retirait les fausses allégations qu'il renfermait (U.S. Ministry of Public Relations, s. d.). En montrant que Cooper aurait soi-disant fait des erreurs dans

18. Ce document contient trente-deux pages de brèves déclarations faites par Cooper (ou des résumés de déclarations particulières), chacune d'elles contredite par la Scientologie. À la suite de ces échanges ont été publiés cinquante-six documents qui, selon des scientologues non identifiés dans l'organisation du ministre des Relations publiques aux ÉtatsUnis, appuyaient leurs réfutations. Par exemple, dans la 11 , l'échange suivant a eu lieu:

SUJET: (p. 71) Un type d'enquête que Hubbard a suggéré était ce qu'il appelait des «enquêtes bruyantes».

FAIT : M. Hubbard n'est pas à l'origine de cette suggestion, et personne n'a été victime de «noisy investigations» selon cette politique qui a été en vigueur pendant plus de six ans (ministre des Relations publiques aux États-Unis, s. d.: 11).

En fait, Hubbard a cependant écrit une lettre de politiques en 1966 au sujet des «attaques contre la Scientologie» et dans une partie examinant un «troisième groupe d'actions [qui] a eu du succès pour arrêter les attaques», Hubbard a mentionné les "noisy investigations» (Hubbard, 1966 a: 491). Sur ce point, Cooper semble avoir eu raison au sujet du fait que Hubbard avait suggéré des «noisy investigations» contre des adversaires. À la page 31, la publication de la Scientologie corrige cependant la référence de Cooper (Cooper, 1971 : 182) qu'elle a faite comme étant PABS (Preclear Auditor's Book), mais dont le titre réel est Professional Auditor's Bulletin (ministre des Relations publique aux États-Unis, s. d.: 31). Cet échange de point/contrepoint se continue sur approximativement deux cents sujets. 
ses recherches, cette correction faisait partie du programme «Dead Agenting» monté contre elle.

Vers 1980, un article "Dead Agenting» moins sophistiqué a circulé parmi les scientologues, et tout indiquait qu'il avait été produit par l'Office du gardien. Il affirmait qu'en 1976, elle avait signé une déclaration avec la Scientologie reconnaissant des erreurs dans son livre et qu'elle se rétractait (Anonymous, 1980?: 13). Bien qu'elle se soit en effet rétractée, elle l'a fait à la suite de harcèlement répété et de procédures judiciaires qui l'avaient épuisée émotionnellement et financièrement (Cité de Clearwater, 1982: 19-20). Larticle «Dead Agenting», moins sophistiqué, transmettait aussi des «faits» tels que «Cooper avait été inculpée pour avoir fait des menaces à la [bombe] par la poste et des faux témoignages» (Ibid.: 10) sans indiquer que la Scientologie elle-même avait fait porter les soupçons sur elle pour ces menaces dans le cadre de l'«Operation Freakout».

De telles tactiques, qui utilisent l'humiliation publique et essaient de briser la personne, reflétaient la rage narcissique que Hubbard avait transposée dans de nombreuses politiques et que les membres du GO appliquaient impitoyablement dans leurs attaques secrètes contre elle. L'utilisation de la politique "Dead Agenting» reflétait aussi le désir narcissique de Hubbard de porter atteinte à Cooper, dans le but de discréditer ses affirmations contre la Scientologie et de faire oublier les accusations publiques qu'elle avait écrites contre lui et son organisation et qui menaçaient son estime de soi.

Quand la Scientologie a identifié des ennemis qu'elle considérait comme des menaces à long terme, elle a intensifié les tactiques de la politique «Dead Agenting» et a mis en application des campagnes «Black $P R$ [relations publiques]». La Scientologie a utilisé la politique confidentielle «Black $P R »$ dans des tentatives pour détruire en fin de compte la réputation de ses ennemis et «pour les discréditer si entièrement qu'ils seront ostracisés» (Hubbard, 1974a: 8). Les campagnes «Black $P R »$ comprenaient des opérations détaillées et à plusieurs aspects destinées à anéantir les détracteurs et à les faire taire pour de bon.

Pendant la décennie suivant la première publication par Cooper de l'article intitulé «The Tragi-Farce of Scientology» (1969), les représailles de la Scientologie se sont intensifiées grâce à l'utilisation d'une campagne «Black $P R$ » contre elle. Le complot de l'organisation contre Cooper a été éventuellement mis à jour grâce à des documents contenus 
dans un fichier intitulé «P.C. OP. FREAKOUT ${ }^{19}$ », que le FBI a découvert lors de descentes aux bureaux de la Scientologie de Washington et de Los Angeles en 1977 (New York Times, 22 janvier 1979) ${ }^{20}$. Ce fichier décrivait les opérations multidimensionnelles du GO pour la faire «interner dans un institut psychiatrique ou incarcérer ou du moins lui faire assez de mal pour qu'elle abandonne ses attaques» ([Guardian's Office], $1^{\text {er }}$ avril 1976).

En plus des actions cohérentes avec les politiques de «Noisy Investigations» et de «Dead Agenting», la Scientologie a utilisé de plus en plus des méthodes créatives et illégales conçues pour l'anéantir et la faire taire une fois pour toutes. Ces méthodes créatives et illégales étaient aussi employées selon les instructions de Hubbard contre des disciples en 1960: «Si attaqué sur quelque point vulnérable par quelqu'un ou quelque chose de n'importe quelle organisation, toujours trouver ou inventer assez de menaces contre eux pour les amener à intenter un procès, afin d'avoir la paix» (Hubbard, 1960: 484). Dans la cause de Cooper, une des plus sérieuses menaces inventées par le GO consistait à faire peser sur elle des soupçons de poseuse de bombes. Après que Cooper eut intenté une poursuite contre la Scientologie, l'organisation a obtenu à son insu ses empreintes et les a placées sur des lettres de menaces à la bombe postées à l'Église de Scientologie de New York. La Scientologie a immédiatement envoyé la preuve fabriquée au FBI, qui à son tour a enquêté sur Cooper. Cooper a coopéré à l'enquête et a été choquée de découvrir que les menaces à la bombe portaient ses empreintes (City of Clearwater, 1982: 11-13 ; New York Times, 22 janvier 1979). Elle a été inculpée sous deux chefs d'accusation du crime d'avoir envoyé des menaces à la bombe par la poste et sous un chef de faux témoignage parce qu'elle niait pendant qu'elle était sous serment (Cooper, 1997: partie 9, page 1). Après avoir subi un interrogatoire après injection de pentothal de sodium, un juge a reporté son procès et lui a ordonné de consulter un psychiatre (City of Clearwater, 1982: 17-18). Deux ans plus tard, une cour a rejeté les accusations portées contre elle.

19. Pour qu'il n'existe aucun malentendu sur ce point, «P.C.» est l'abréviation de «Paulette Cooper» et «OP.» d'«opération».

20. Voir: Cour de district des États-Unis pour le district central de la Californie, 1978: 170 : «Cedars Inventory, 11972-74, sujet 46 - Dossier manille format commercial nommé "Op. Freakout" contenant trois documents». 
Une autre tactique «Dead Agenting» incluait des efforts pour anéantir Cooper émotionnellement et perturber ses relations avec sa famille et ses amis. Des scientologues de Boston ont cambriolé le bureau de son ancien psychiatre, enlevé et photocopié son dossier avant de le remettre en place (Bradlee, 1983: 4). Des membres de la Scientologie ont ensuite posté des copies de son dossier à ses parents et amis avec comme résultat qu'« elle a subi un grave traumatisme émotionnel, perdu du poids, était incapable de travailler et souffrait de paranoïa» (United States District Court for the District of Massachusetts, 1981: 3).

Les tactiques de la Scientologie utilisées contre Cooper suivaient les politiques que Hubbard avait conçues et qui reflètent son «comportement agressif, sadique, vengeur» (Ronningstam, 2005: 110) associé aux gens atteints de la forme maligne de la névrose narcissique. La capacité de la Scientologie de "participer à des activités criminelles» (Malmquist, 1996: 165) montre aussi dans quelle mesure les membres de l'organisation étaient prêts à satisfaire la rage narcissique de Hubbard. La campagne «Black $P R$ » contre Cooper illustre en fin de compte la propension de Hubbard à «mettre en œuvre des plans pour anéantir une autre personne, souvent celle qui représente une embûche pour l'empêcher d'obtenir encore plus de gloire» (Malmquist, 1996: 165).

Bien que ces politiques du GO fassent la démonstration du narcissisme malin de Hubbard, la politique «Auditing Process R2-45» est l'ultime manifestation de sa rage narcissique. Révélée la première fois dans The Creation of Human Ability, en 1954, la politique «Auditing Process R2-45» y était décrite comme «un processus énormément efficace pour l'extériorisation ${ }^{21}$, mais son utilisation est mal vue par la société en ce moment» (Hubbard, 1954: 120). «Un certain nombre d'anciens scientologues qui sont maintenant des détracteurs de l'organisation affirment que R2-45 a été conçue pour autoriser à tuer ses adversaires avec un pistolet de calibre 45.» (Rawitch et Gillette, 28 août 1978: 2)

Les scientologues ne tiennent pas compte de la légitimité de la politique, mais la documentation interne de l'organisation fait la preuve de l'existence de «Auditing Process R2-45». Dans un ordre éthique intitulé «Racket Exposed», Hubbard dresse la liste de douze "personnes suppressives» qu'il déclare «ennemies de l'humanité, de la planète et

21. «L'extériorisation, dans la terminologie de la Scientologie, est la capacité de l'esprit ou "thétan" de physiquement quitter le corps» (Rawitch et Gillette, 28 août 1978: 2). 
de toute vie». Il les a aussi identifiées comme devant être traitées par la politique «Fair Game» et a donné les instructions que «[t]out membre de Sea Org qui communique avec n'importe lequel d'entre eux devait utiliser "Auditing Process R2-45"» (Hubbard, 1968b: 1). Il a reproduit l'ordre éthique (et a ajouté un nom à la liste) dans un journal de l'organisation appelé The Auditor, que les scientologues recevaient partout dans le monde (Hubbard, 1968c). Plus tard la même année (1968), Hubbard a imprimé un autre ordre R2-45 dans The Auditor, cette fois contre quatre autres personnes (Hubbard, 1968d). Il n'existe pas de preuve précise qu'un membre de Sea Org ait suivi les instructions meurtrières de Hubbard, mais un incident comprenant un parent de Paulette Cooper vaut la peine d'être raconté.

Quand Cooper a témoigné, en 1982, aux audiences de la City of Clearwater Commission concernant l'Église de Scientologie, elle a affirmé que la Scientologie avait inclus «Auditing Process R2-45» dans ses tactiques contre elle. Elle a révélé qu'elle avait déménagé dans un appartement plus sécuritaire en 1972, et que sa cousine, Joy, qui lui ressemble physiquement, avait pris le sien. Quatre jours après le déménagement, Joy a répondu à la porte pour apercevoir un homme tenant un bouquet de fleurs dissimulant un fusil. Il a sorti son arme, l'a pointée vers sa tête et a appuyé sur la gâchette. Le fusil ne s'est pas déchargé et l'individu a commencé à l'étrangler jusqu'à ce qu'elle se mette à crier. Il s'est ensuite enfui. Cooper a par la suite témoigné sous serment qu'elle croyait être la personne visée par l'attaque, qu'elle considérait comme une tentative ratée de la tuer, et que l'incident était une application d'«Auditing Process R2-45» (City of Clearwater, 1982: 10; voir Cooper, 1997: Partie 3:2) 22 .

22. Une déclaration faite en 1994 par l'ancienne membre Hana Whitfield (anciennement Eltringham), dont nous avons parlé plus tôt, rapporte une autre allégation d'une opération «R 2-45» ratée. Exposée à une accusation de parjure, elle a déclaré que pendant qu'elle se trouvait à Bizerte, en Tunisie (Afrique du Nord): «Deux officiers du Sea Org et moi parlions sur le quai un soir. Ils prenaient tous les deux l'avion pour Los Angeles le lendemain, une mission confiée par Hubbard. Ils m’ont révélé que Hubbard leur avait ordonné de tirer sur Jack Horner, qui vivait à Los Angeles. Hubbard l'avait lui-même déclaré personne répressive quelques années auparavant. Les deux hommes avaient des fusils qu'ils apporteraient durant le voyage. Ils sont partis... et sont revenus quelques semaines plus tard. Après leur compte-rendu, ils m'ont affirmé que Horner avait en effet été victime de coups de feu à plusieurs reprises à sa résidence, un soir, alors qu'il se trouvait en compagnie de sa famille. Heureusement, personne n'avait été blessé.» (Whitfield, 1994: 19) 
«Auditing Process R2-45» est de façon dramatique la manifestation du narcissisme malin de Hubbard et plus précisément de sa rage narcissique. Le fait qu'il ait conçu et autorisé cette politique démontre que «selon la dynamique du meurtre narcissique, tuer constitue un acte vertueux de représailles, un effort désespéré pour obtenir le contrôle et pour sauvegarder et augmenter l'estime de soi» (Ronningstam, 2005: 107; voir Malmquist, 1996). La mise en application probable de la politique contre Cooper illustre la rage narcissique qui prend des proportions meurtrières (Malmquist, 1996: 168) et se situe près de la limite extrême d'un continuum de réactions possibles à des menaces à l'estime de soi de Hubbard. De plus, la nature impitoyable et l'envergure des campagnes de la Scientologie contre ses ennemis sont une indication de la volonté de quelques disciples de Hubbard de faire taire les personnes dont les affirmations et les actions sont considérées comme menaçantes par lui.

\section{Conclusion}

La théorie criminologique n'a pas encore tiré parti de la quantité considérable de matériel que les sectes, les cultes et les nouvelles religions utilisent - qui sont tous des termes que les chercheurs ont appliqués à la Scientologie au cours des années. À l'intérieur de ces groupes existe une grande variété de comportements qui souvent impliquent la déviance et la criminalité (Robbins, 1988), ce qui diffère peu des comportements semblables observés dans certains gouvernements et certaines entreprises et que les criminologues ont analysés longuement. Les comportements criminels dans quelques-unes de ces sectes vont $\mathrm{du}$ délit international de grande envergure de l'organisation aux mouvements mondiaux de personnel et de ressources dans leurs efforts pour commettre ou couvrir des crimes. En fait, les crimes religieux ou sectaires, qui sont des actions illégales «perpétrées en accord avec les buts opérationnels des groupes», comprennent tous les types de délits observés dans les analyses criminologiques traditionnelles (Kent, 1998: 121-122), même si on en fait rarement mention, par exemple, dans les analyses de crimes des cols blancs (Lane, 2005; cf. Barnhill, 2005).

Puisque les criminologues découvrent cette abondance d'informations dont l'examen a été négligé, ils vont profiter de l'attention portée au rôle crucial joué par les fondateurs et les chefs dans la plupart des 
actions criminelles dans lesquelles de nombreuses sectes s'engagent. De plus, ils seront en accord avec des collègues du milieu des affaires qui sont au courant des activités dysfonctionnelles et parfois criminelles des chefs narcissiques depuis au moins deux décennies (voir, par exemple, Schwartz, 1987; Sankowsky, 1995 ; Maccoby, 2000; Lubit, 2002). En toute probabilité, la connaissance du trouble narcissique dans le monde des affaires pourra s'appliquer directement à l'étude criminologique des sectes, puisque de nombreuses sectes exploitent des entreprises d'une complexité considérable. Une recherche semblable a été réalisé par la communauté des analystes politiques, qui ont examiné des sujets comme le narcissisme malin et la rage narcissique chez les tyrans politiques (Post, 1993: 109, 113-114; Glad, 2002: 2-25) ainsi que la relation dysfonctionnelle entre les chefs charismatiques narcissiques et leurs disciples (Post, 1986).

Il est étonnant, cependant, que les sociologues de la religion aient résisté à l'enrichissement de leurs propres approches par des observations psychologiques et psychiatriques. Des batailles hégémoniques qui durent depuis longtemps entre la sociologie et la psychologie sur les désignations d'expertise expliquent probablement en partie cette résistance. Les sociologues ont une aversion profondément enracinée pour l'interprétation des phénomènes sociaux par des concepts psychologiques. De plus, quelques chercheurs en sociologie ont en horreur d'aborder la criminalité commise par les sectes et les nouvelles religions, parce qu'ils ont peur que des informations critiques au sujet de ces groupes ne nuisent aux efforts anti-cultes.

Par exemple, le sociologue David Bromley et l'historien des religions Gordon Melton ont minimisé l'influence que les chefs dysfonctionnels exercent sur les groupes sectaires en concluant: «Attribuer les résultats d'une organisation à la personnalité d'un seul individu, même d'un puissant chef charismatique, camoufle habituellement une dynamique sociale beaucoup plus complexe.» (Melton et Bromley, 2002: 47) Dans notre analyse sur Hubbard, cependant, l'identification du narcissisme malin dévoile, plutôt qu'il ne camoufle, la condition psychologique sous-jacente qui a fourni le contexte à la «dynamique sociale complexe» des représailles des membres contre les détracteurs et les personnes perçues comme ennemies. Il émerge de notre analyse des questions sociales cruciales concernant la socialisation des membres pour qu'ils perçoivent le monde comme Hubbard - un sujet qui nécessiterait une étude exhaustive. 
Lorne Dawson est un autre sociologue très en vue qui diminue l'importance des interprétations psychologiques (mais nous préférons biopsychosociales) des chefs de secte. Dans un article dans lequel il se demande si les psychopathologies jouent un rôle dans le charisme et la violence associés aux nouvelles religions, il conclut qu'«il n'existe pas de preuve suffisante pour prétendre que les leaders charismatiques sont de quelque façon plus enclins que les autres personnes à un comportement violent ou à élaborer des politiques qui conduisent à la violence» (Dawson, 2006: 21). Il ajoute:

Je suis peu porté à suivre l'hypothèse de presque toutes les analyses psychologiques sur les leaders charismatiques, à savoir qu'ils souffrent d'une certaine forme identifiable de névrose ou même de psychose. Nous n'avons tout simplement pas les données requises [sic] pour en venir à une telle conclusion de façon certaine. (Dawson, 2006: 21)

Au moins si nous prenons le cas de L. Ron Hubbard, il est tout à fait évident que «la personnalité d'un seul individu» constitue la variable principale nécessaire pour expliquer les politiques déviantes et parfois criminogènes de la Scientologie, et que la preuve de son narcissisme malin est accablante.

\section{Références}

American Psychiatric Association (2000a). Hypomanic Episode. Diagnostic and Statistical Manual of Mental Disorders ( $4^{\text {th }}$ ed.). Washington, D. C.: American Psychiatric Association. Disponible en ligne.

- (2000b). 301.81 Narcissistic Personality Disorder. Diagnostic and Statistical Manual of Mental Disorders (4 ${ }^{\text {th }}$ ed.). Washington, D. C.: American Psychiatric Association. Disponible en ligne.

Anderson, R. D. (1999). Inside the Mind of Joseph Smith: Psychobiography and the Book of Mormon. Salt Lake City: Signature Books.

Anonymous (1980?). Paulette Cooper. [Probable Guardian's Office Dead Agent Pack]. Manuscrit non publié. In Stephen A. Kent Collection on Alternative Religions. Housed in the University of Alberta Library. Accessible en ligne.

Armstrong, G. (1984a). Reporters' Transcript of Proceedings. Superior Court of the State of California for the County of Los Angeles. Church of Scientology of California vs. Gerald Armstrong, Mary Sue Hubbard, Intervenor, C 420153: 1289-1563.

- (1984b). Reporters' Transcript of Proceedings. Superior Court of the State of California for the County of Los Angeles. Church of Scientology of Califor- 
nia vs. Gerald Armstrong, Mary Sue Hubbard, Intervenor, C 420153: 17401895.

Atack, J. (1990). A Piece of Blue Sky: Scientology, Dianetics, and L. Ron Hubbard Exposed. Secaucus, New Jersey: Lyle Stuart.

Bainbridge, W. S., \& Stark, R. (1979). Cult Formation: Three Compatible Models. Sociological Analysis, 40, 283-295. Réédition in R. Stark \& W. S. Bainbridge (1985), The Future of Religion: Secularization, Revival and Cult Formation (171-188). Berkeley: University of California Press.

Barker, E. (1984). The Making of a Moonie: Choice or Brainwashing? Oxford: Basil Blackwell.

Barnhill, J. (2005). Religious Fraud. In L. M. Salinger (ed.), Encyclopedia of White-Collar \& Corporate Crime (2, 679-685). London: Sage.

Bradlee Jr., B. (31 mai 1983). Scientology Defectors Charge 'Dirty Tricks' in Boston. Boston Globe. Consulté le $1^{\text {er }}$ juin 2007, <http://www.xenu.net/ archive/go/media/bg310583.htm>

Brodie, F. M. (1963). No Man Knows My History: The Life of Jospeb Smith. New York: Alfred A. Knopf.

Burden, T. (25 janvier 1980). Affidavit. Las Vegas, Nevada.

Carter, L. F. (1990). Charisma and Control in Rajneeshpuram: The Role of Shared Values in the Creation of a Community. Cambridge: Cambridge University Press.

City of Clearwater (1982). Commission Hearing: The Church of Scientology. Day 4, Paulette Cooper. May 8. Consulté le 31 mai 2007, <www.xenu-directory. net/documents/cw1982/cw4_314-348.htm >

Clark, R. O. (1988). The Narcissistic Guru: A Profile of Bhagwan Shree Rajneesh. Free Inquiry, printemps, 33-45.

Clarke, J. (9 septembre 1950). Noted Doctors Attack New Treatment. Daily News [Los Angeles, California], p. 3, 16.

Clarke, P. (2006). New Religions in Global Perspective: A Study of Religious Change in the Modern World. London: Routledge.

Cohn, N. (1970). The Pursuit of the Millennium (édition de 1957 révisée et élargie). New York: Oxford University Press.

Cooper, P. (1969). The Tragi-Farce of Scientology. Queen Magazine [London]. Consulté le 29 janvier 2008, <http://www.cs.cmu.edu/ dst/Library/ Shelf/cooper/sos-que.html>

- (1971). The Scandal of Scientology. New York: Tower Books.

- (1997). Paulette Cooper's Harassment Diary. Accessible en ligne < http://www. holysmoke.org/pc/pc.htm>

Corydon, B. (1996). L. Ron Hubbard: Messiab or Madman? (Édition de 1992 révisée). Fort Lee, New Jersey: Barricade Books.

Dawson, L. (2006). Psychopathologies and the Attribution of Charisma: A Critical Introduction to the Psychology of Charisma and the Explanation of Violence in New Religious Movements. Nova Religio, 10 (2), 2-28. 
Deutsch, A. (1983). Psychiatric Perspectives on an Eastern-Style Cult. In D. A. Halperin (ed.), Psychodynamic Perspectives on Religion Sect and Cult (113-129). Boston: John Wright.

- (1989). Psychological Perspectives on Cult Leadership. In M. Galanter (ed.), Cults and New Religious Movements: A Report of the American Psychiatric Association (147-163). Washinton, D. C.: American Psychiatric Association.

Glad, B. (2002). Why Tyrants Go Too Far: Malignant Narcissism and Absolute Power. Political Psychology, 23 (1), 1-37.

Glock, C. (1964). The Role of Deprivation in the Origin and Evolution of Religious Groups. In R. Lee \& M. E. Martin (eds.), Religion and Social Conflict (24-36). New York: Oxford.

[Guardian's Office]. (1er avril 1976). Operation Freakout. Document Obtained During the FBI's July 8-9, 1977 Raid on Scientology Offices in Washington, D.C. and Los Angeles.

Hubbard, L. R. (1950). Dianetics: The Modern Science of Mental Health. New York: Hermitage House.

- (1951). Handbook for Preclears. [Sans lieu]: Scientific Press.

- (1954). The Creation of Human Ability. Sussex, England: Department of Publications Worldwide.

- (1955). The Scientologist: A Manual on the Dissemination of Material. In L. R. Hubbard (1976c), 151-171.

- (1956a). The Problems of Work. Washington, D. C. : Scientology Consultants to Industrial Efficiency.

- (1956b). Scientology: the Fundamentals of Thought. London: Hubbard Association of Scientologists International.

- (1959). Manual of Justice. Confidential - for HCO Personnel Only. London: Hubbard Communications Office.

- (1960). Dept of Govt Affairs. Hubbard Communications Policy Letter (August 15). In L. R. Hubbard, 1974c: 483-485.

- (1965a). Ethics. Suppressive Acts. Suppression of Scientology and Scientologists. The Fair Game Law. Hubbard Communications Office Policy Letter (December 23). In L. R. Hubbard (1970). The Organization Executive Course (1, 552-557). Copenhagen: Scientology Publications Organization.

- (1965b). Suppressive Persons, Main Characteristics Of. Hubbard Communications Office Policy Letter (August 7). In L. R. Hubbard (1070), The Organization Executive Course (1, 428-430). Copenhagen: Scientology Publications Organization.

- (1966a). Attacks on Scientology. Hubbard Communications Office Policy Letter (February 18). In L. Ron Hubbard, 1974c: 491-493.

- (1966b). The Guardian. Hubbard Communications Office Policy Letter (March 1).

- (1967a). Penalties for Lower Conditions. Hubbard Communications Office Policy Letter (October 18). 
- (1967b). Post, Handling Of. Hubbard Communication Office Policy Letter (September 12). In L. R. Hubbard (1991). The Organization Executive Course, Basic Staff Hat, (0, 238-239). Los Angeles: Bridge Publications.

- (1968a). Cancellation of Fair Game. Hubbard Communications Office Policy Letter (October 21). In L. R. Hubbard (1970). The Organization Executive Course, HCO Division 1. (1 ed., 489). Copenhagen: Scientology Publications Organization.

- (1968b). Racket Exposed. Hubbard Communications Office Ethics Order, 30 INT, (March 6).

- (1968c). Racket Exposed. The Auditor, 35. East Grinstead: Saint Hill Manor.

- (1968d). Racket Exposed. The Auditor, 37. East Grinstead: Saint Hill Manor.

- (1968e). The War. Executive Directive from L. Ron Hubbard, 55 INT (November 29).

- (1969). Organization Program No.1. LRH Executive Directive 49 INT (9 December). In L. R Hubbard (1970). The Organization Executive Course, Basic Staff, (0, 256-260). Copenhagen: Scientology Publications Organization.

- (1972). How to Handle Black Propaganda. Hubbard Communications Office Policy Letter (November 21). In L. R. Hubbard (1982), The Management Series (2, 418-427). Los Angeles: Bridge Publications.

- (1974a). Handling Hostile Contacts/Dead Agenting. Bodrd Policy Letter, Confidential PR Series 24 (May 30).

- (1974b). The Technical Breakthrough of 1973! The Introspection RD. Hubbard Communications Office Bulletin (January 23). In L. R.Hubbard (1976c: 239-242).

- (1974c.) The Organization Executive Course (7, 1976, $2^{\text {th }}$ ed.) Los Angeles: Church of Scientology of California.

- (1976a). Modern Management Technology Defined. Copenhagen: New Era Publications.

- (1976b). The Technical Bulletins of Dianetics and Scientology (I, 1950-1953). Copenhagen: Scientology Publications.

- (1976c). The Technical Bulletins of Dianetics and Scientology (II, 1972-1975). Copenhagen: Scientology Publications.

- (1976d). The Technical Bulletins of Dianetics and Scientology, (VIII, 1972-1975). Copenhagen: Scientology Publications.

- (1982). The Future of Scientology. Ron's Journal 34 (March 13).

James, W. (1902). The Varieties of Religious Experience. New York: The Modern Library/Random House.

Kent, S. A. (1987a). Psychological and Mystical Interpretations of Early Quakerism: William James and Rufus Jones. Religion, 17 : 251-274.

- (1987b). Psychology and Quaker Mysticism: the Legacy of William James and Rufus Jones. Quaker History, 76 (1), 1-17. 
- (1987c). Puritan Radicalism and the New Religious Organizations: Seventeenth Century England and Contemporary America. Comparative Social Research, 10, 3-46. Greenwich, Connecticut: JAI Press.

- (1989). Mysticism, Quakerism, and Relative Deprivation: A Sociological Reply to R.A. Naulty. Religion, 19: 157-178.

- (1998). Crimes. In W. H. Swatos (ed.), Encyclopedia of Religion and Society (121-122). London: Altamira Press.

- (1999). The Globalization of Scientology: Influence, Control and Opposition in Transnational Markets. Religion, 29, 147-169.

- (2001). From Slogans to Mantras: Social Protest and Religious Conversion in the Late Vietnam War Era. Syracuse: Syracuse University Press.

- (2006). Scientific Evaluation of the Dangers Posed by Religious Groups: A Partial Model. In P. Côté \& T. J. Gunn (eds.), The New Religious Question: State Regulation or State Interference? (343-370). Brusselles: Peter Lang. Revised Version Previously Published in 2004, Cultic Studies Review 3 (2 \& 3), 101-134.

- (2007). Narcissistic Fraud in the Ancient World: Lucian's Account of Alexander of Abonuteichos and the Cult of Glycon. Ancient Narrative, 6 : 77-99.

Kernberg, O. F. (1975). Borderline Conditions and Pathological Narcissism $\left(2^{\text {th }}\right.$ ed., 1976). New York: Jason Aronson.

- (1984). Severe Personality Disorders: Psychotherapeutic Strategies (édition de 1986). New Haven: Yale University Press.

- (1992). Aggression in Personality Disorders and Perversions. New Haven: Yale University Press.

- (1998). Pathological Narcissism and Narcissistic Personality Disorder: Theoretical Background and Diagnostic Classification. In E. F. Ronningstam (ed.), Disorders of Narcissism: Diagnostic, Clinical, and Empirical Implications (29-51). Washington, American Psychiatic Press.

Kohut, H. (1972). Thoughts on Narcissism and Narcissistic Rage. The Psychodnalytic Study of the Child, 27, 360-400. New York: Quadrangle Books.

Krakauer, J. (2003). Under the Banner of Heaven: A Story of Violent Faith. New York: Doubleday.

Kumar, J. P. 1997. "Fair Game": Leveling the Playing Field in Scientology Litigation. Review of Litigation, 16 (3), 747-772.

Lalich, J. (2004). Bounded Choice: True Believers and Charismatic Cults. Berkeley: University of California Press.

Lane, J. M. (2005). The Guardian's Office of Scientology: A Test of James Coleman's Theory of Elite Deviance. M.A. Thesis, Department of Sociology, University of Alberta, Alberta.

Lieberman, A. R. (2004). The Narcissistic Personality Disorder. In J. F.Masterson $\&$ A. R. Lieberman (eds.), A Therapist's Guide to the Personality Disorders: The Masterson Approach (73-90). Phoenix, Arizona: Zeig, Tucker \& Theisen.

LRH Aides (1969). Covert Operations. LRH Aides' Conference (Novembre 2 1969). Conférence à bord du navire Apollo, $31 \mathrm{p}$. 
Lubit, R. (2002). The Long-Term Organizational Impact of Destructively Narcissistic Managers. Academy of Management Executive, 16 ( 1), 127-138.

Lys, C. (2005). The Violence of Jim Jones: A Biopsychosocial Explanation. Cultic Studies Review, 4 (3), 267-294.

Maccoby, M. (2000). Narcissistic Leaders: The Incredible Pros, the Inevitable Cons. Harvard Business Review (January-February) : 69-77.

Malmquist, C. P. (1996). Homicide: A Psychiatric Perspective. Washington, DC: American Psychiatric Press.

Mascareñas de los Santos, C., \& Mascareñas Ruiz, J. (1997). Un estudio psicoanalítico sobre la relación líder-feligresía en la Iglesia "La Luz del Mundo." In "La Luz del Mundo". Un análisis multidisciplinario de la controversia religiosa que ba impactado a nuestro país (85-116). Édité par Revista académica para el estudio de las religiones. Mexico: Estudio científico de las religiones.

Melton, J. G., \& Bromley, D. G. (2002). Challenging the Misconceptions About the New Religions - Violence Connection. In D. G.. Bromley \& J. G. Melton (eds.), Cults, Religion and Violence (42-56). Cambridge: Cambridge University Press.

Miller, R. (1987). Bare-Faced Messiab : The True Story of L. Ron Hubbard. Toronto: Key Porter Books.

Mullins, M. R. (1997). Aum Shinrikyo as an Apocalyptic Movement. In T. Robbins \& S. J. Palmer (eds.), Millennium, Messiabs, and Maybem: Contemporary Apocalyptic Movements (313-324). London: Routledge.

National Parole Board (2003). NPB Pre-Release Decision Sheet [on Ivon Shearing]. (April 15), 5p.

Newport, K. G. C. (2006). The Branch Davidians of Waco: The History and Beliefs of an Apocalyptic Sect. Oxford: Oxford University Press.

New York Times (22 janvier 1979). Author of a Book on Scientology Tells of Her 8 Years of Torment., p. A-14.

Numbers, R. L., \& Numbers, J. S. (1992). Afterward: Ellen White on the Mind and the Mind of Ellen White. In R. L. Numbers (ed.), Prophetess of Health: Ellen G. white and the Origins of Seventh-day Adventist Health Reform (202-227). Knoxville: University of Tennessee Press.

Osinski, B. (2007). Ungodly: A True Story of Unprecedented Evil. Macon, Georgia: Indigo Publishing Group.

Phillips, K. A., Yen, S., \& Gunderson, J. G. (2008). Personality Disorders: Narcissistic Personalyty Disorder. In R. E. Hales \& S. C. Yudofsky (eds.). Textbook of Clinical Psychiatry (4 ${ }^{\mathrm{e}}$ édition, 2pp.). Arlington, Virginia: American Psychiatric Publishing. Accessible en ligne.

Post, J. M. (1986). Narcissism and the Charismatic Leader-Follower Relationship. Political Psychology, 7 (4 ), 675-688.

- (1993). Current Concepts of the Narcissistic Personality: Implications for Political Psychology. Political Psychology, 14 (1), 99-121.

Raine, S.. (2005).Reconceptualizing the Human Body: Heaven's Gate and the Quest for Divine Transformation Religion, 35: 98-117. 
Rawitch, R., \& Gillette, R. (28 août 1978). Scientology Critics Assail Aggressiveness of Church. Los Angeles Times, <http://www.xenu.net/archive/go/ media/la280878.htm>.

Robbins, T. (1988). Profit for Prophets: Legitimate and Illegitimate Economic Practices in New Religious Movements. In J. T. Richardson (ed.), Money and Power in the New Religions (69-116). Queenston, Ontario: The Edwin Mellen Press.

Ronningstam, E. F. (2005). Identifying and Understanding the Narcissistic Personality. Oxford: Oxford University Press.

Ronningstam, E., \& Gunderson, J. (1990). Identifying Criteria for Narcissistic Personality Disorder. American Journal of Psychiatry, 147 (7), 918-922.

Sankowsky, D. (1995). The Charismatic Leader as Narcissist: Understanding the Abuse of Power. Organizational Dynamics, 23 (Spring): 57-71.

Schwartz, H. S. (1987). On the Psychodynamics of Organizational Totalitarianism. Journal of Management, 13 (1), 41-54.

Sil, N. P. (1991). Ramakrsna Paramahamsa: A Psychological Profile. Leiden: E. J. Brill.

Singer, M. T., \& Lalich, J. (1995). Cults in Our Midst. San Francisco: JosseyBass.

Storr, A. (1996). Feet of Clay: Saints, Sinners, and Madmen: A Study of Gurus. Toronto: The Free Press.

Supreme Court of the State of New York (1972). Paulette Cooper, Plaintiff, against Church of Scientology of New York, Inc.; Church of Scientology of California; and U.S. Churches of Scientology, Defendants. Verified Amended Complaint., Index \# 6732/72 (June 21). County of New York: Supreme Court of the State of New York. Consulté le 1 janvier 2007,

$<$ http://groups.google.com/groups? $\mathrm{q}=\% 22 \mathrm{Church}+\mathrm{of}+$ Scientology + of + California $\% 22+\% 22$ Queen $\% 22+\% 22$ Paulette + Cooper $\% 22 \&$ hl $=$ en $\& 1 \mathrm{r}=\&$ ie $=U T F-8 \&$ oe $=$ UTF- $8 \&$ edition $=$ us $\&$ selm $=58 \mathrm{c} 6 \mathrm{co} \% 24 \mathrm{~d} 74 \% 40 \mathrm{clark}$. zippo.com \& rnum $=2>$

United States District Court for the Central District of California (1978). Church of Scientology of California v. United States of America. Respondent's Submission of Search Warrants Inventories Marked with Documents to be Returned to Petitioner. No. CV 77-2565-MML (April 4).

United States District Court For the District of Columbia (1979a). Stipulation of Evidence. United States of America vs. Mary Sue Hubbard, et. al. Criminal 78401 (October 26).

United States District Court For the District of Columbia (1979b). Sentencing Memorandum of the United States of America. United States of America vs. Mary Sue Hubbard, et. al. Criminal 78-401 (December 3).

United States District Court for the District of Massachusetts (1981). Paulette Cooper, Plaintiff, vs. Church of Scientology of Boston, Inc., L. Ron Hubbard and Mary Sue Hubbard, Defendants. Complaint. (March 9).

U. S. Ministry of Public Relations (s.d., circa 1974). False Report Correction: The Scandal of Scientology by Paulette Cooper. [Los Angeles?]: Church of Scientology of California. 
Wallis, R. (1977). The Road to Total Freedom: A Sociological Analysis of Scientology. New York: Columbia University Press.

Weber, M. (1922) [1968]. Economy and Society (2 vols.). In G. Roth \& C. Wittich (eds.), (Trad. E. Fischoff, et. al.) Réédition, 1978. Berkeley: University of California Press.

Whitfield, H. (1994). Revised Declaration of Hand Whitfield Re Motion for Costs. United States District Court, Central District of California, "Church of Scientology International vs. Steven Fishman and Uwe Geertz." CV 91-6426 HLH (Tx). (March 8).

Williams, C. L. (1951). Special Orthopedic Examination [of Lafayette R. Hubbard]. [August 1, 2 p.]. Veterans Administration Center. Witchita, Kansas.

Wilson, B. 1970. Religious Sects: A Sociological Study. Toronto: McGraw Hill Book Company.

Wilson, M. (1993). DSM III and the Transformation of American Psychiatry: A History. American Journal of Psychiatry, 150 (3), 399-410.

ABSTRACT - In this article, a personality disorder known as "malignant narcissism" is presented. This notion is then used to explain the creation of organizational policies against perceived enemies that reflected this narcissistic rage. We illustrate our argument by the analysis of a case study in which it is shown that the leader attempted to discredit the detractors of the group, thus transposing the narcissistic rage into organizational policies that loyal members enacted on his behalf. By using psychological insights about the leader's personality, and then showing how that personality translated into socially deviant policies and actions, we hope to encourage criminologists to examine other groups by applying similar theories.

RESUMEN - En este artículo presentamos el trastorno de la personalidad denominado "narcisismo maligno". Este concepto es a continuación utilizado para explicar la creación, por parte del dirigente de un grupo, de políticas organizacionales destinadas a contrarrestar a las personas consideradas por él como enemigas y que ponen de manifiesto su ira narcisista. Nuestra argumentación es examinada a la luz de un estudio de caso en el que se demuestra que el dirigente intentó desacreditar a los detractores del grupo, trasfiriendo su ira narcisista a políticas organizacionales que fueron adoptadas por miembros leales a la organización. Gracias a observaciones psicológicas de la personalidad del líder y, más adelante, demostrando cómo su personalidad está en la base de políticas y acciones socialmente desviantes, esperamos alentar a los criminólogos a examinar otros grupos aplicando teorías similares. 